University of Nebraska - Lincoln

DigitalCommons@University of Nebraska - Lincoln

February 1995

\title{
FAR-ULTRAVIOLET STELLAR PHOTOMETRY: FIELDS IN SAGITTARIUS AND SCORPIUS
}

\author{
Edward G. Schmidt \\ University of Nebraska-Lincoln, eschmidt1@unl.edu \\ George R. Carruthers \\ E.O. Hulburt Center for Space Research, Naval Research Laboratory, Washington, DC
}

Follow this and additional works at: https://digitalcommons.unl.edu/physicsschmidt

Part of the Physics Commons

Schmidt, Edward G. and Carruthers, George R., "FAR-ULTRAVIOLET STELLAR PHOTOMETRY: FIELDS IN SAGITTARIUS AND SCORPIUS" (1995). Edward Schmidt Publications. 16.

https://digitalcommons.unl.edu/physicsschmidt/16

This Article is brought to you for free and open access by the Research Papers in Physics and Astronomy at DigitalCommons@University of Nebraska - Lincoln. It has been accepted for inclusion in Edward Schmidt Publications by an authorized administrator of DigitalCommons@University of Nebraska - Lincoln. 
The ASTRophysical Journal SuPPLEMENT SeRIES, 96:605-626, 1995 February

(1) 1995. The American Astronomical Society. All rights reserved. Printed in U.S.A.

\title{
FAR-ULTRAVIOLET STELLAR PHOTOMETRY: FIELDS IN SAGITTARIUS AND SCORPIUS
}

\author{
EDWARD G. SCHMIDT \\ Division of Astronomical Sciences, National Science Foundation, 4201 Wilson Boulevard, Arlington, VA 22230; eschmidt@unlinfo.unl.edu \\ AND \\ GEORGE R. CARRUTHERS \\ E.O. Hulburt Center for Space Research, Code 7609, Naval Research Laboratory, Washington, DC 20375-5320 \\ Received 1994 May 20; accepted 1994 August 10
}

\begin{abstract}
Far-ultraviolet photometry for 741 objects in a field in Sagittarius centered near M8 and 541 objects in a field centered near $\zeta$ Scorpii is presented. These data were extracted from electrographic images obtained with two cameras during a shuttle flight in 1991 April/May. The cameras provided band passes with $\lambda_{\text {eff }}=1375 \AA$ and $\lambda_{\mathrm{cff}}=1781 \AA$. Synthetic colors show that these bands are sensitive to effective temperature for hot stars. Our measurements were placed on a quantitative far-ultraviolet magnitude scale by convolving the spectra of stars observed by $I U E$ with our cameras' spectral response functions. Fifty-eight percent of the ultraviolet objects were identified with visible stars using the SIMBAD database while another $40 \%$ of the objects are blends of early type stars too close together to separate with our resolution. Our photometry is compared with that from the $T D-1$, $O A O 2$, and $A N S$ satellites and the S201 (Apollo 16) far-ultraviolet camera and found to agree at the level of a few tenths of a magnitude. Unlike previous studies, almost half of the identified visual counterparts to the ultraviolet objects are early B stars. A plot of distance modulus against ultraviolet color excess reveals a significant population of stars with strong ultraviolet excesses.
\end{abstract}

Subject headings: stars: early-type - surveys — techniques: photometric — ultraviolet: stars

\section{INTRODUCTION}

Previous papers have reported ultraviolet stellar photometry obtained with electrographic cameras operated on the lunar surface by Apollo 16 astronauts (Experiment S201; Page, Carruthers, \& Heckathorn 1982; Carruthers \& Page, 1983, $1984 a, b, c)$ or carried aboard sounding rockets (Schmidt \& Carruthers 1993a, hereafter Paper I), and 1993b, hereafter Paper II).

Two NRL Far-Ultraviolet Cameras were operated in Earth orbit during Space Shuttle Mission STS-39 from 1991 April 28 to May 6. Images were obtained of 12 star fields. This paper presents stellar photometry for two of those fields, one centered in Sagittarius and one centered in Scorpius. The Sagittarius field overlaps one observed with a very similar camera during the Apollo 16 mission (Page et al. 1982; Carruthers \& Page 1984b).

\section{THE INSTRUMENTATION}

The camera fields were circular with a diameter slightly less than $20^{\circ}$. For the observations reported here, Camera 1 was used with a $\mathrm{CaF}_{2}$ filter. This provided sensitivity between 1220 and $1620 \AA$ and an effective wavelength for flat photon flux of $1375 \AA$ A. Camera 2 with a $\mathrm{SiO}_{2}$ filter was sensitive between 1610 and $2000 \AA$ with effective wavelength of $1781 \AA$. Further details regarding the cameras can be found in papers by Carruthers (1986) and Carruthers et al. (1992). Details of the camera calibrations, including pre-flight and post-flight measurements are given by Carruthers et al. (1994) and Christensen et al. (1994).
To explore the properties of the far ultraviolet cameras' photometric system, magnitudes were calculated using fluxes from the new Kurucz model atmospheres (described by Kurucz 1993 and distributed on Kurucz CD-ROM No. 13) and the preflight response functions from Carruthers (1986). All the models were constructed with a microturbulent velocity of 2.0 $\mathrm{km} \mathrm{s}^{-1}$. We have only used models with solar composition but have included all gravities and all temperatures above $6000 \mathrm{~K}$.

We combined the calculated ultraviolet magnitudes with Kurucz's tabulated $V$-magnitudes to form the colors $\left(m_{1375}-\right.$ $\left.m_{1781}\right)$ and $\left(m_{1781}-V\right)$. In doing this we set the zero point so that $m_{\mathrm{uv}}=10.0$ and $V=10.0$ correspond to a flux of $F_{\lambda}=$ $3.6 \times 10^{-13}$ ergs $\mathrm{cm}^{-2} \mathrm{~s}^{-1} \AA^{-1}$. This is equivalent to $m_{\lambda}=-2.5 \log F_{\lambda}-21.1$.

The calculated colors are listed in Tables 1 and 2 and are plotted in Figure 1. Both colors show significant gravity dependence. The $\left(m_{1781}-V\right)$ index retains its temperature sensitivity throughout the entire range but $\left(m_{1375}-m_{1781}\right)$ is insensitive to temperature above about $20,000 \mathrm{~K}$ and below about $6750 \mathrm{~K}$.

\section{THE OBSERVATIONS AND ANALYSIS}

Table 3 lists the images used in this paper. One field was centered near M8 in Sagittarius while the other was centered near $\zeta$ Sco. The wide range of exposure times provides for a large dynamic range. In Figure 2 (Plates 14-17) we present prints from both fields taken with both cameras.

Following the same procedures as described in Paper I the images were digitized with the NRL PDS microdensitometer, 
TABLE 1

Calculated $\left(m_{1375}-m_{1781}\right)$

\begin{tabular}{|c|c|c|c|c|c|c|c|c|c|c|}
\hline$T_{\text {eff }} / \log g$ & 0.50 & 1.00 & 1.50 & 2.00 & 2.50 & 3.00 & 3.50 & 4.00 & 4.50 & 5.00 \\
\hline $6250 \ldots \ldots \ldots$ & 6.34 & 6.51 & 6.66 & 6.75 & 6.84 & 6.90 & 6.93 & 6.93 & 6.89 & 6.82 \\
\hline $6750 \ldots \ldots \ldots$ & 6.21 & 6.37 & 6.54 & 6.70 & 6.84 & 6.71 & 6.79 & 6.85 & 6.89 & 6.89 \\
\hline $7000 \ldots \ldots \ldots . .$. & 6.17 & 6.28 & 6.43 & 6.56 & 6.66 & 6.75 & 6.54 & 6.60 & 6.65 & 6.67 \\
\hline $7250 \ldots \ldots \ldots$ & 5.83 & 6.01 & 6.16 & 6.29 & 6.38 & 6.45 & 6.52 & 6.27 & 6.34 & 6.39 \\
\hline $7500 \ldots \ldots \ldots . .$. & 4.71 & 5.19 & 5.41 & 5.60 & 5.78 & 5.93 & 6.06 & 6.16 & 5.93 & 6.02 \\
\hline $8250 \ldots \ldots \ldots . . .$. & $\ldots$ & 1.72 & 2.17 & 2.53 & 2.89 & 3.27 & 3.64 & 4.03 & 4.44 & 4.73 \\
\hline $8500 \ldots \ldots \ldots . . . .$. & $\ldots$ & $\ldots$ & 1.43 & 1.65 & 1.90 & 2.23 & 2.62 & 3.05 & 3.55 & 4.05 \\
\hline $8750 \ldots \ldots . . . . .$. & $\ldots$ & $\ldots$ & 1.01 & 1.10 & 1.24 & 1.44 & 1.74 & 2.12 & 2.58 & 3.17 \\
\hline $9000 \ldots \ldots \ldots . . .$. & $\ldots$ & $\ldots$ & 0.77 & 0.77 & 0.82 & 0.94 & 1.13 & 1.41 & 1.79 & 2.26 \\
\hline $9250 \ldots \ldots \ldots$ & $\ldots$ & $\ldots$ & $\ldots$ & 0.57 & 0.57 & 0.62 & 0.74 & 0.93 & 1.21 & 1.59 \\
\hline $9500 \ldots \ldots \ldots \ldots$ & $\ldots$ & $\ldots$ & $\ldots$ & 0.44 & 0.40 & 0.41 & 0.48 & 0.61 & 0.81 & 1.10 \\
\hline $9750 \ldots \ldots \ldots . . . . . . . .$. & $\ldots$ & $\ldots$ & $\ldots$ & 0.35 & 0.29 & 0.27 & 0.30 & 0.38 & 0.53 & 0.75 \\
\hline $12500 \ldots \ldots \ldots$ & $\ldots$ & $\ldots$ & $\ldots$ & $\ldots$ & -0.15 & -0.20 & -0.23 & -0.25 & -0.25 & -0.24 \\
\hline $13000 \ldots \ldots . .$. & $\ldots$ & $\ldots$ & $\ldots$ & $\ldots$ & -0.19 & -0.24 & -0.27 & -0.29 & -0.29 & -0.29 \\
\hline $14000 \ldots \ldots \ldots$ & $\ldots$ & $\ldots$ & $\ldots$ & -0.17 & -0.26 & -0.31 & -0.35 & -0.37 & -0.37 & -0.37 \\
\hline $15000 \ldots \ldots \ldots$ & $\ldots$ & $\ldots$ & $\ldots$ & $\ldots$ & -0.31 & -0.37 & -0.41 & -0.43 & -0.44 & -0.44 \\
\hline $16000 \ldots \ldots \ldots . . . . . .$. & $\ldots$ & $\ldots$ & $\ldots$ & $\ldots$ & -0.35 & -0.42 & -0.46 & -0.48 & -0.49 & -0.49 \\
\hline $17000 \ldots \ldots \ldots$ & $\ldots$ & $\ldots$ & $\ldots$ & $\ldots$ & -0.38 & -0.46 & -0.50 & -0.53 & -0.54 & -0.55 \\
\hline $18000 \ldots \ldots . . .$. & $\ldots$ & $\ldots$ & $\ldots$ & $\ldots$ & -0.41 & -0.49 & -0.54 & -0.57 & -0.58 & -0.59 \\
\hline $19000 \ldots \ldots \ldots$ & $\ldots$ & $\ldots$ & $\ldots$ & $\ldots$ & -0.43 & -0.52 & -0.57 & -0.61 & -0.62 & -0.63 \\
\hline $20000 \ldots \ldots \ldots$ & $\ldots$ & $\ldots$ & $\ldots$ & $\ldots$ & $\ldots$ & -0.54 & -0.60 & -0.63 & -0.66 & -0.67 \\
\hline $21000 \ldots \ldots \ldots$ & $\ldots$ & $\ldots$ & $\ldots$ & $\ldots$ & $\ldots$ & -0.56 & -0.62 & -0.66 & -0.69 & -0.70 \\
\hline $22000 \ldots \ldots \ldots$ & $\ldots$ & $\ldots$ & $\ldots$ & $\ldots$ & $\ldots$ & -0.57 & -0.64 & -0.69 & -0.71 & -0.73 \\
\hline $23000 \ldots \ldots \ldots$ & $\ldots$ & $\ldots$ & $\ldots$ & $\ldots$ & $\ldots$ & -0.58 & -0.66 & -0.71 & -0.74 & -0.75 \\
\hline $24000 \ldots \ldots \ldots$ & $\cdots$ & $\ldots$ & $\ldots$ & $\cdots$ & $\ldots$ & -0.59 & -0.67 & -0.73 & -0.76 & -0.78 \\
\hline $25000 \ldots \ldots \ldots$ & $\ldots$ & $\ldots$ & $\ldots$ & $\ldots$ & $\ldots$ & -0.59 & -0.68 & -0.74 & -0.77 & -0.80 \\
\hline $35000 \ldots \ldots \ldots$ & $\ldots$ & $\ldots$ & $\ldots$ & $\ldots$ & $\ldots$ & $\ldots$ & $\ldots$ & -0.72 & -0.78 & -0.82 \\
\hline $37500 \ldots \ldots \ldots$ & $\ldots$ & $\ldots$ & $\ldots$ & $\ldots$ & $\ldots$ & $\ldots$ & $\ldots$ & $\ldots$ & -0.78 & -0.82 \\
\hline 40000 & $\ldots$ & $\ldots$ & $\ldots$ & $\ldots$ & $\ldots$ & $\ldots$ & $\ldots$ & $\ldots$ & -0.78 & -0.82 \\
\hline $42500 \ldots \ldots . . . . .$. & $\ldots$ & $\ldots$ & $\ldots$ & $\ldots$ & $\ldots$ & $\ldots$ & $\ldots$ & $\ldots$ & $\ldots$ & -0.82 \\
\hline $45000 \ldots \ldots \ldots$. & $\ldots$ & $\ldots$ & $\ldots$ & $\ldots$ & $\ldots$ & $\ldots$ & $\ldots$ & $\ldots$ & $\ldots$ & -0.82 \\
\hline $47500 \ldots \ldots \ldots$ & $\ldots$ & $\ldots$ & $\ldots$ & $\ldots$ & $\ldots$ & $\ldots$ & $\ldots$ & .. & $\ldots$ & -0.82 \\
\hline $50000 \ldots \ldots \ldots$ & $\ldots$ & $\ldots$ & $\ldots$ & $\ldots$ & $\ldots$ & $\ldots$ & & & $\ldots$ & -0.83 \\
\hline
\end{tabular}

stars were found using the Stetson (1987) method (and verified visually) and instrumental magnitudes were extracted by aperture photometry using IRAF. In the M8 field 741 objects were found while the $\zeta$ Sco field yielded 541 objects. These objects are listed in Tables 4 and 5. Tables 4 and 5 will also be made available in machine-readable form in the AAS CD-ROM series, Vol. 4.

Fifty-six stars in the $\zeta$ Sco field and 84 stars in the M8 field were identified in the SAO catalog and a polynomial fit was made between the image coordinates and the celestial coordinates. The rms scatter in these fits were between 1.0 and 1.3.
This can be taken to be the accuracy of our coordinates which are listed for the individual stars in the second and third columns of Tables 4 and 5.

The FWHM of the star images was 5.0 for Camera 1 and 6.4 for Camera 2. Unlike the images analyzed in Papers $I$ and II, the FWHM was reasonably constant from the field center to the edge. Thus, the correction for location in the field which was applied previously was unnecessary (that is to say, $a$ in eq. [2] (2) of Paper I is zero). This resulted in a better determination of the saturation corrections.

For each star we formed a weighted mean magnitude from 
TABLE 2

Calculated $\left(m_{1781}-V\right)$

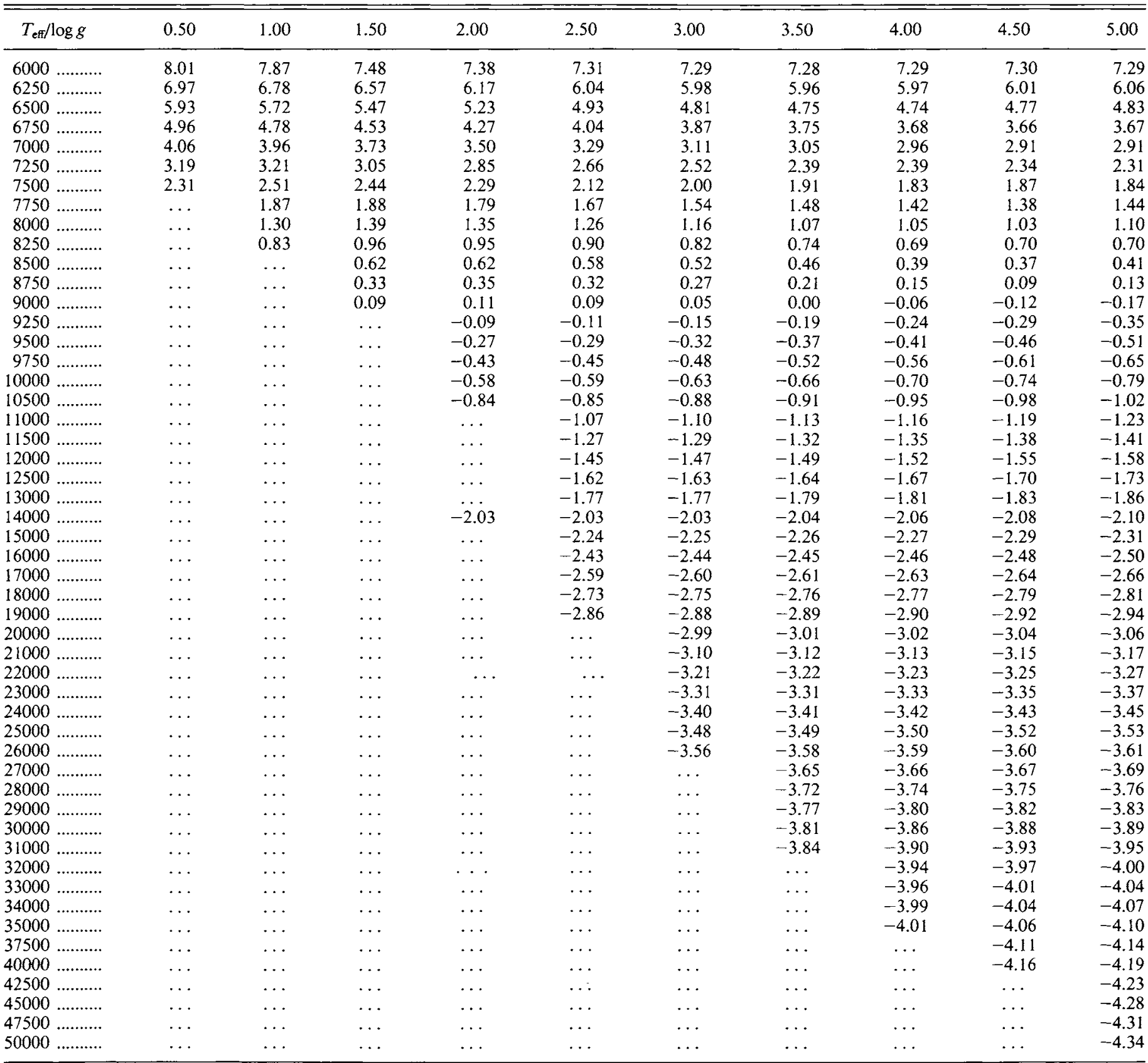

all the images on which it was measured. Although magnitudes from images which are saturated at the center are corrected as described in Paper I, the correction introduces uncertainty. Hence, progressively lower weight was given to images with greater saturation; only the magnitudes of a few of the brightest stars will be sensitive to the saturation correction. Similarly, lower weights were given to star images which were poorly exposed. Again, only the faintest stars will have magnitudes which depend on weak images.

We identified a number of stars for which $I U E$ spectra are available. After eliminating those where blending was suspected (based largely on identifications from SIMBAD as discussed below) the spectra were convolved with the preflight sensitivity functions (Carruthers 1986) to obtain the ultraviolet magnitudes. The zero point corrections to place our magnitudes on the standard system (as defined above with $m_{\mathrm{uv}}=10.0$ at a flux of $F_{\lambda}=3.6 \times 10^{-13} \mathrm{ergs} \mathrm{cm}^{-2} \mathrm{~s}^{-1} \AA^{-1}$ ) were then determined. Although the actual sensitivities of the cameras as a function of wavelength are still being determined (Carruthers et al. 1994), only the shape of the response curves (not their 
TABLE 3

LOG OF OBSERVATIONS

\begin{tabular}{ccccccc}
\hline \hline Field & Date & Time & $\alpha_{1950}$ & $\delta_{1950}$ & Camera & \multicolumn{1}{c}{ Exposure } \\
\hline M8 ............ & 1991 May 5 & $6: 40$ & $18^{\mathrm{h}} 01^{\mathrm{m}}$ & $-24^{\circ} 35^{\prime}$ & 1 & $3,10,30,100,300$ \\
& & & & & 2 & $3,10,30,100$ \\
$\zeta$ Sco .......... & 1991 May 4 & $23: 45$ & $16^{\mathrm{h}} 56^{\mathrm{m}}$ & $-41^{\circ} 10^{\prime}$ & 1 & $3,10,30,30,100$ \\
& & & & & 2 & $3,10,30,30$ \\
\hline
\end{tabular}

absolute values) are significant since the $I U E$ absolute spectrophotometry is used to calibrate our observations. The results are only weakly dependent on slight deviations of the relative sensitivity versus wavelength curves from the preflight measurements. For the magnitudes from Camera $1\left(m_{1375}, 20\right.$ stars), the rms scatter was 0.22 magnitudes and thus the zero point has a standard error of 0.05 magnitudes. For Camera 2 ( $m_{1781}, 16$ stars), the rms scatter was 0.26 magnitudes and the standard error of the mean was 0.06 magnitudes. The ultraviolet magnitudes corrected with these zero points are listed in the fourth and fifth columns of Tables 4 and 5 .

The use of $I U E$ fluxes to calibrate our magnitudes is subject to some uncertainty due to the degredation of the IUE cameras. An examination of the dates of the $I U E$ spectra indicates that the degredation is unlikely to affect our zero points at a level any greater than the standard errors cited above. When
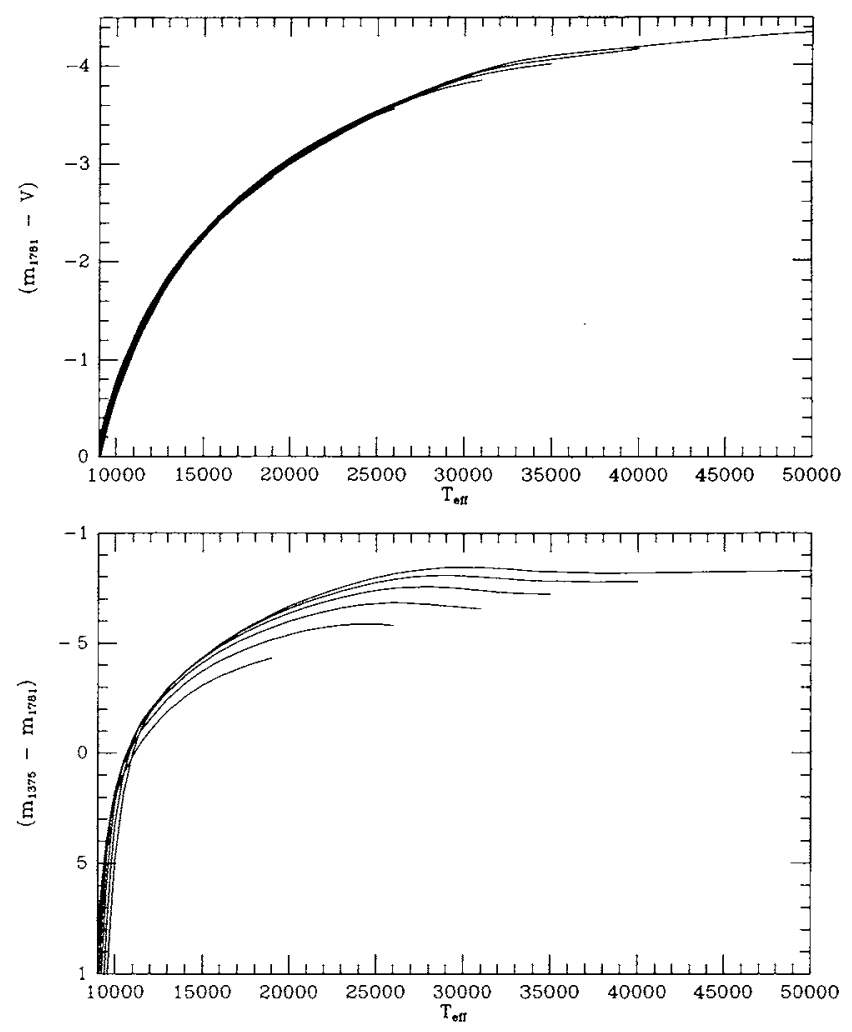

FIG. 1.-Calculated ultraviolet colors for Kurucz models. The various curves represent $\log g=2.5,3.0,3.5,4.0,4.5$, and 5.0 from the lowest to the uppermost. all of the far-ultraviolet camera images have been analyzed and the IUE final archive is complete, we intend to rediscuss the ultraviolet magnitude calibration.

The scatter in the comparison of our instrumental magnitudes with those from IUE spectra is a combination of measurement errors for individual stars, sensitivity variations across the camera fields and uncertainties in the $I U E$ fluxes. Assuming the latter is about $10 \%$ ( $\mathrm{T}$. Teays 1994, private communication ), we can attribute total errors of 0.22 magnitudes to our photometry for stars in the brightness range of the IUE calibration stars, $3.5<m_{\text {uv }}<8$.

We interrogated the SIMBAD database for stars near the ultraviolet objects. As in previous papers, we have given the information for stars which are possibly associated with the ultraviolet objects in the fifth, sixth, and seventh columns of Tables 4 and 5 . When there is more than one star which can plausibly contribute to the ultraviolet flux the identification is given as "Blend," a composite $V$ or $P$ magnitude is given and the spectral types of all the stars are listed in order of optical brightness. For objects associated with a star cluster, the spectral type refers to the dominant star in the group if there is one.

In the present fields $58 \%$ of the ultraviolet objects are identified with visible stars. Forty percent are blends while 22 objects are identified with star clusters or parts of star clusters. Only eight objects were not identified with any known object from the SIMBAD database.

\section{COMPARISON WITH OTHER PHOTOMETRY}

The TD-1 (Thompson et al. 1978), OAO 2 (Code, Holm, \& Bottemiller 1980), ANS (Wesseluis et al. 1982) and S201 (Page et al. 1982) catalogs were searched for stars in common with Tables 4 and 5. Only stars with unique visible identifications were included.

In Figure 3 we compare $m_{1781}$ with the magnitudes from the previous studies which matched it most closely in effective wavelength. It can be seen that both the $A N S$ and the $T D-1$ magnitudes are systematically fainter than ours. The differences are $0.05 \mathrm{mag}$. and $0.29 \mathrm{mag}$. for $m_{1565}$ and $m_{1965}$ from $T D-1$ with rms scatter about these values of $0.56 \mathrm{mag}$. The $A N S$ 18 and $15 \mathrm{~W}$ magnitudes are 0.02 and 0.11 mag fainter than ours on average with rms scatter of 0.73 and $0.53 \mathrm{mag}$. On the other hand, except for several of the brightest stars, the $O A O 2$ $m_{1919}$ magnitudes tend to be fainter than $m_{1781}$, while the $m_{1550}$ magnitudes are brighter. The mean differences for both are 0.28 and $0.42 \mathrm{mag}$ with scatter of 0.23 and $0.29 \mathrm{mag}$.

Aside from these offsets, the stars closely parallel the solid lines for stars with $m_{1781}$ in the range from about four to eight. Fainter than this the $T D-I$ magnitudes tend to be somewhat 


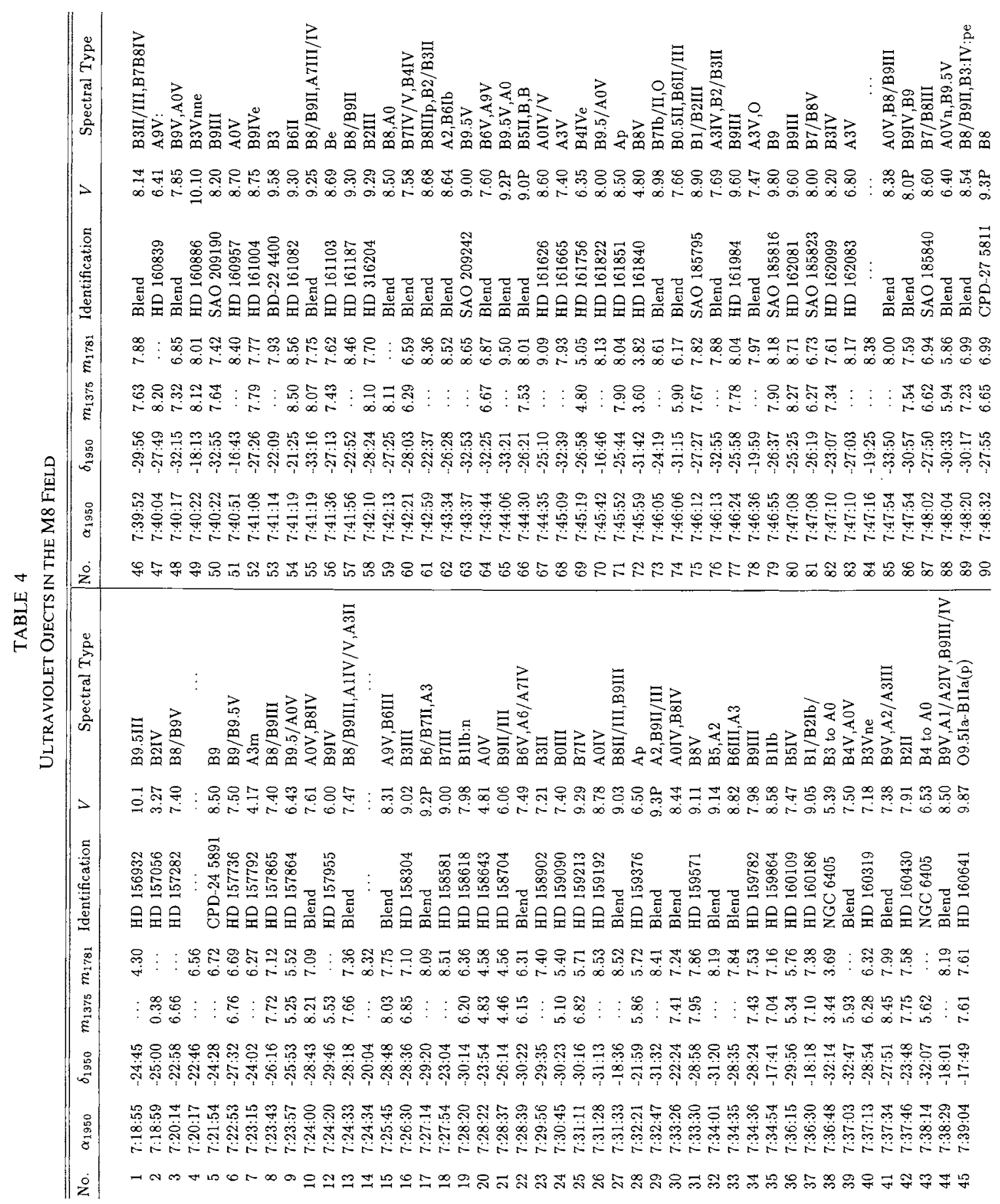

(C) American Astronomical Society - Provided by the NASA Astrophysics Data System 


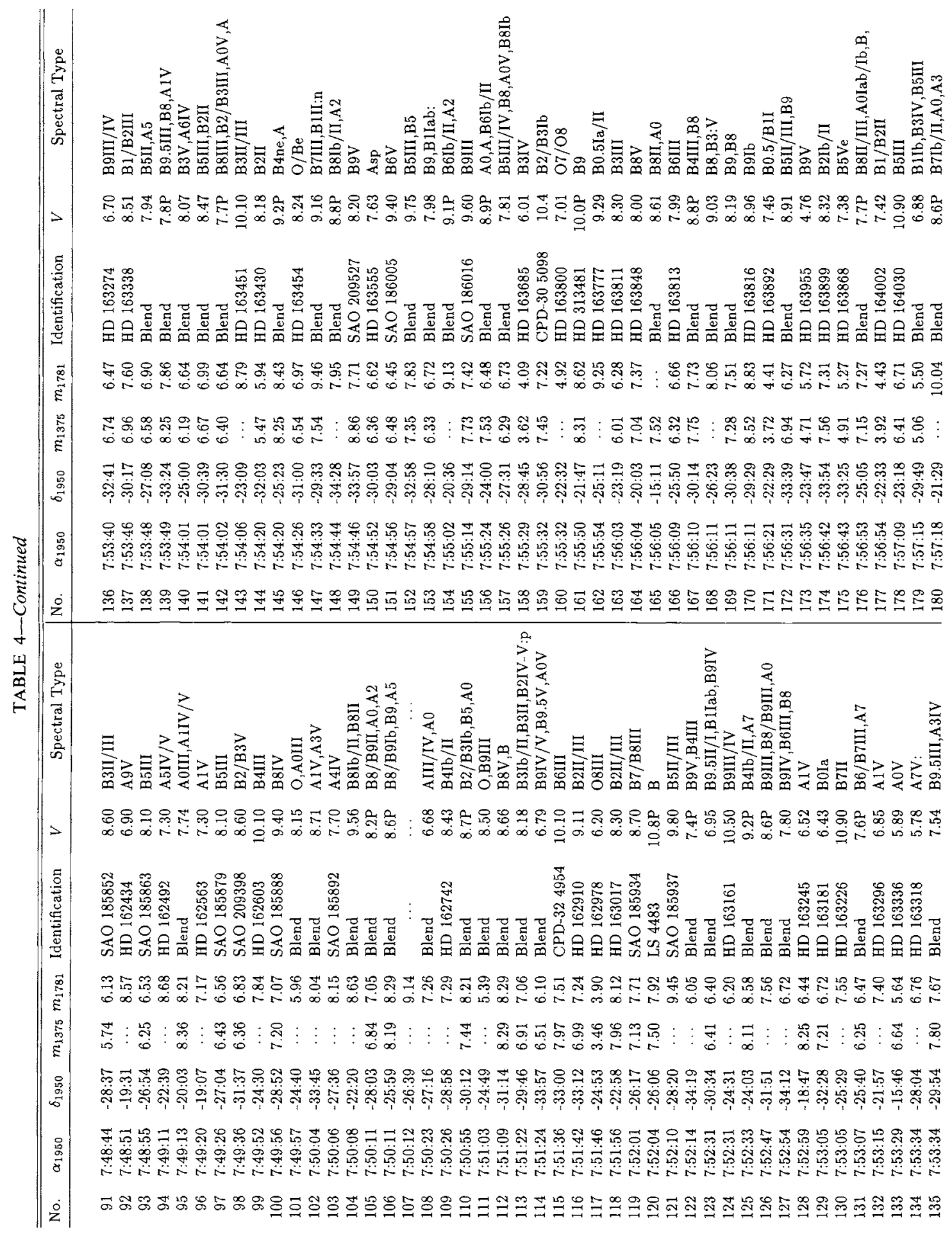

610

(C) American Astronomical Society - Provided by the NASA Astrophysics Data System 


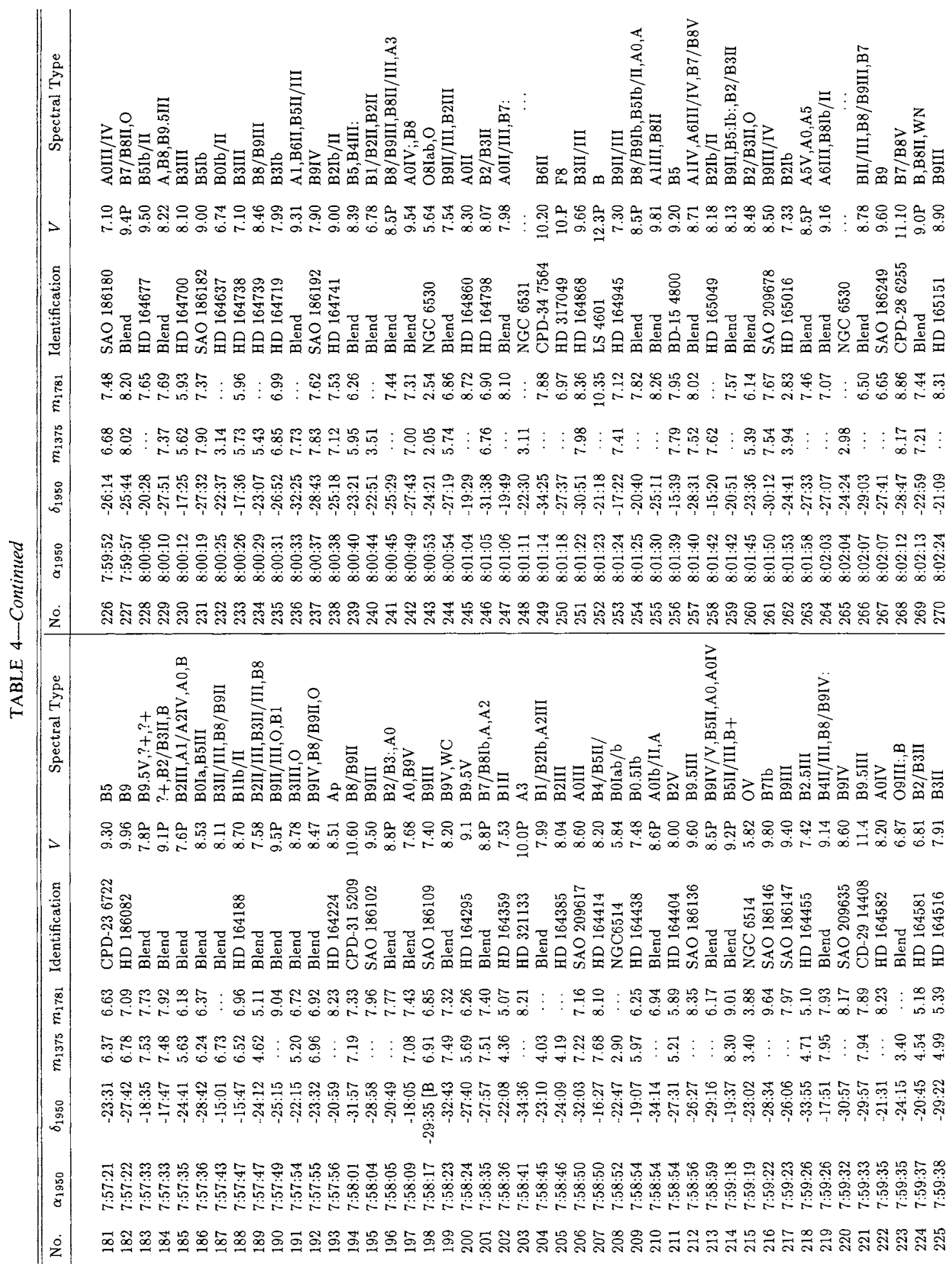




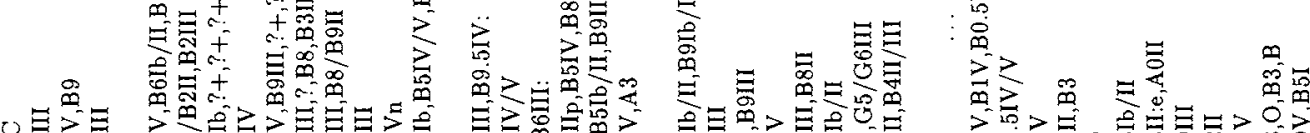

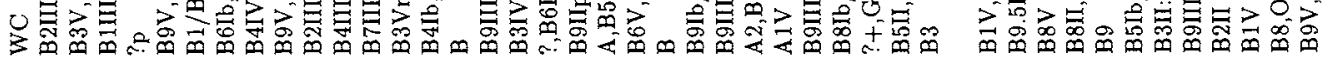

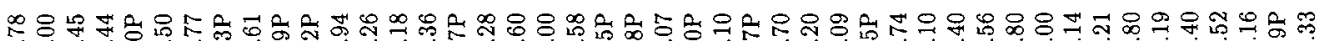

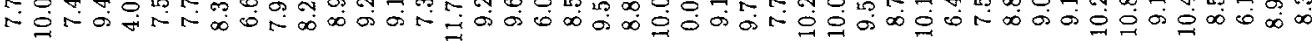

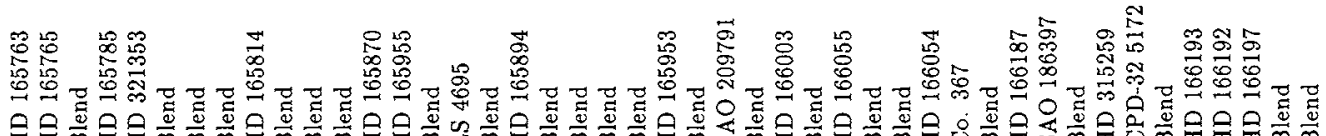

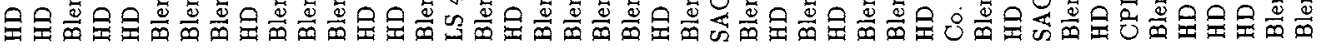

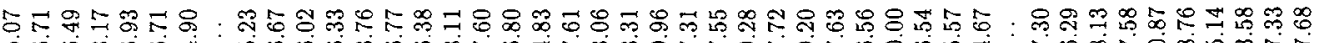
获

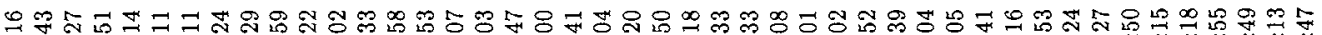

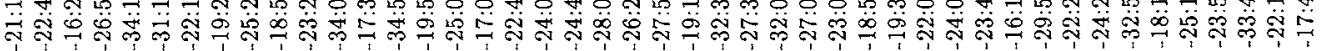

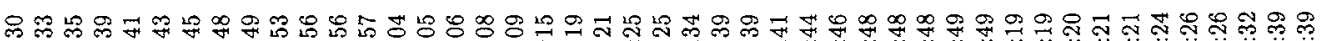

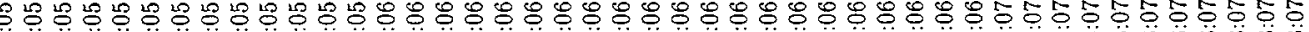

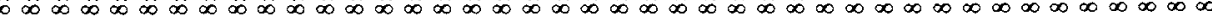

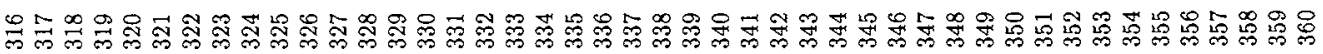

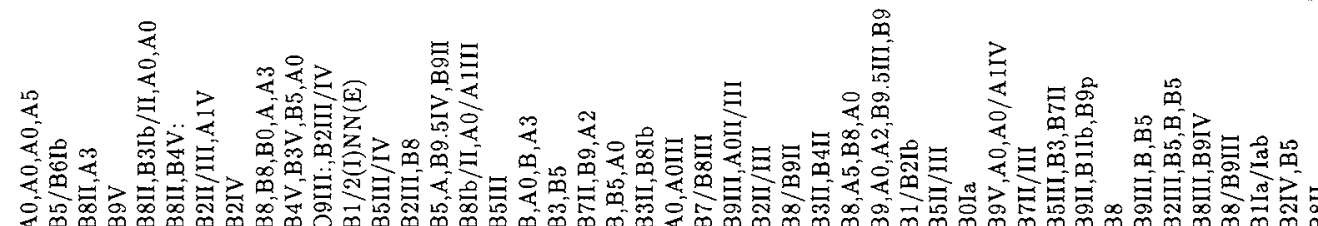

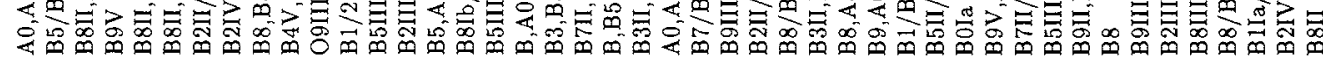

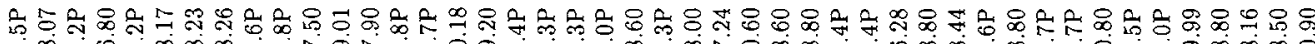

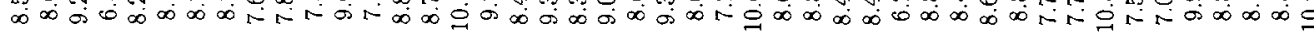

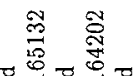

突

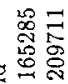

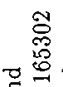

篗

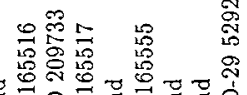

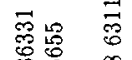

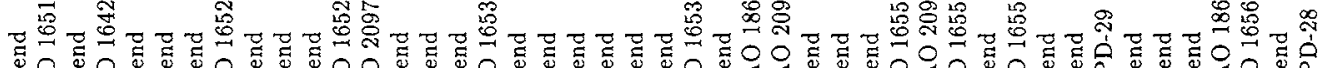

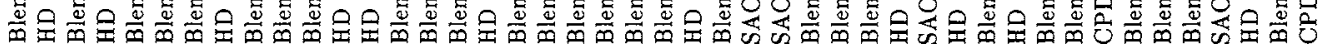

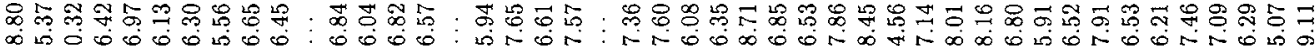

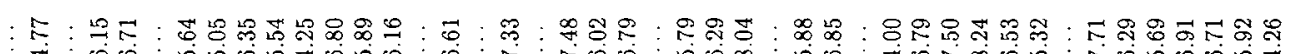

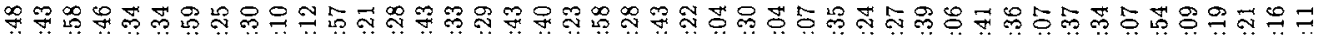

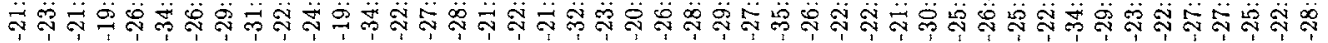

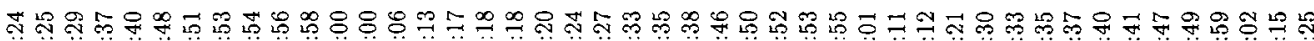
ㄱㅇㅇ

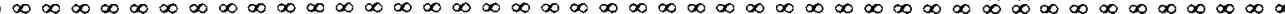

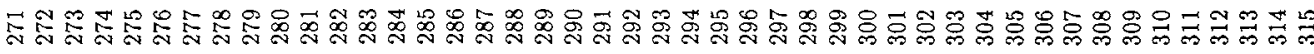




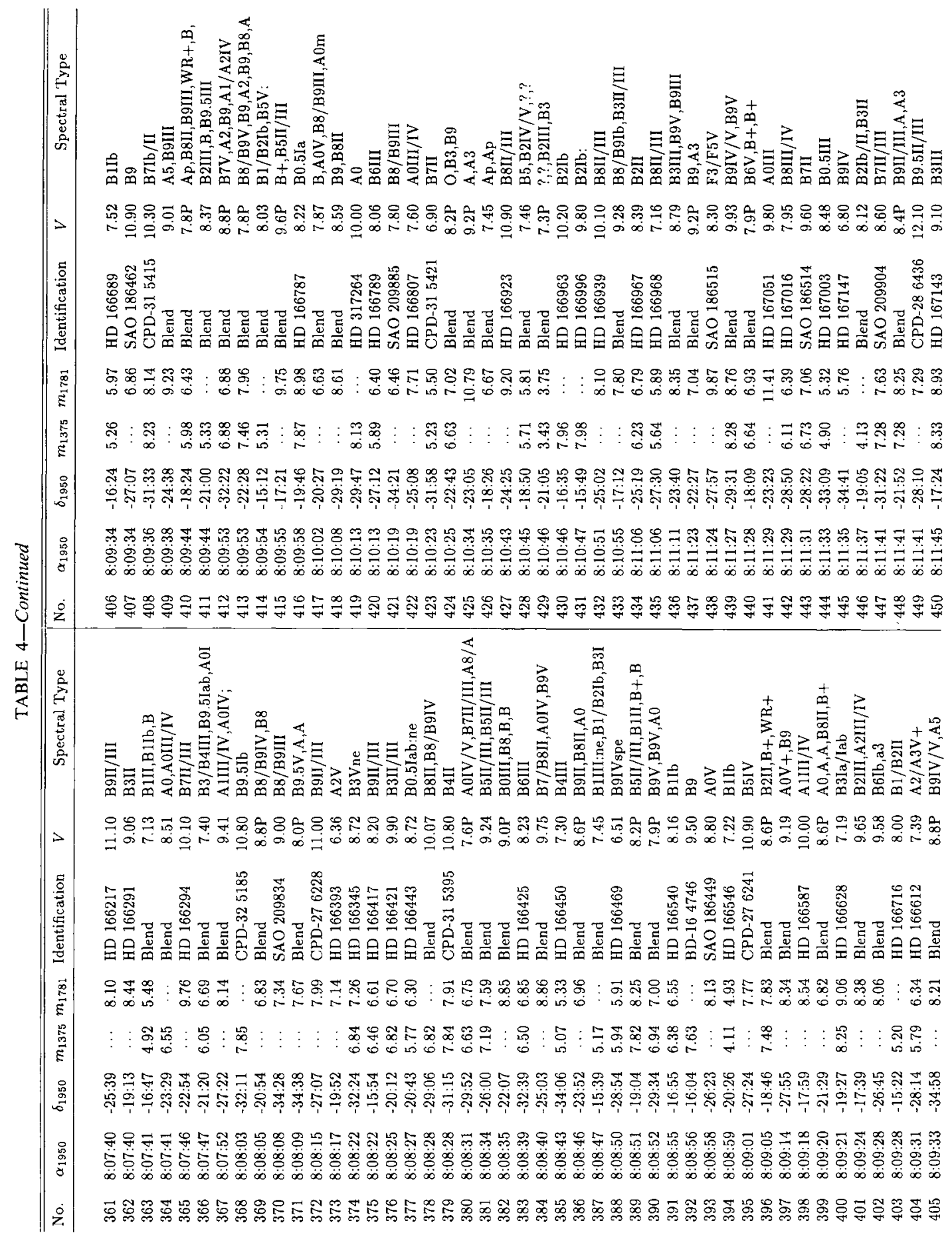




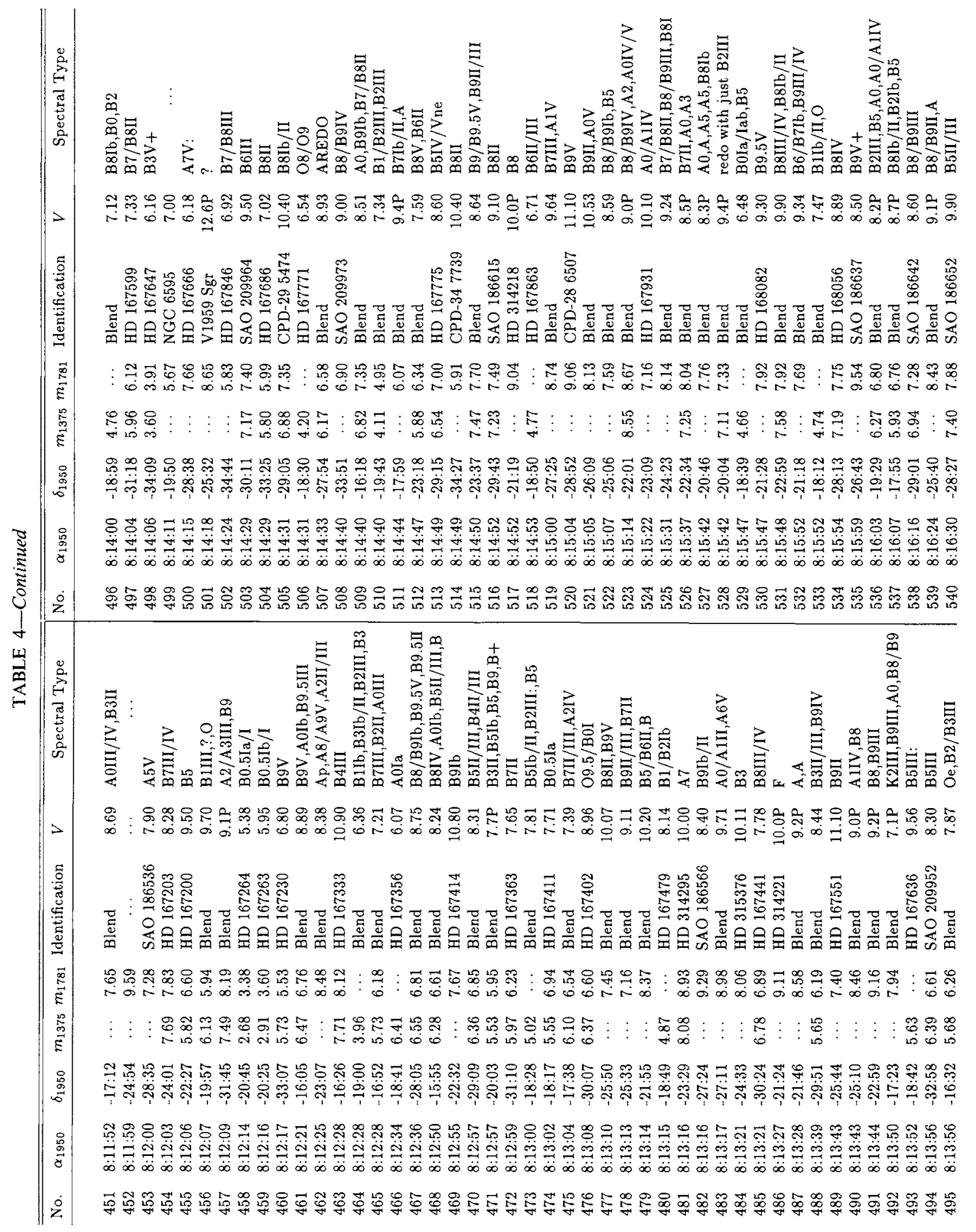




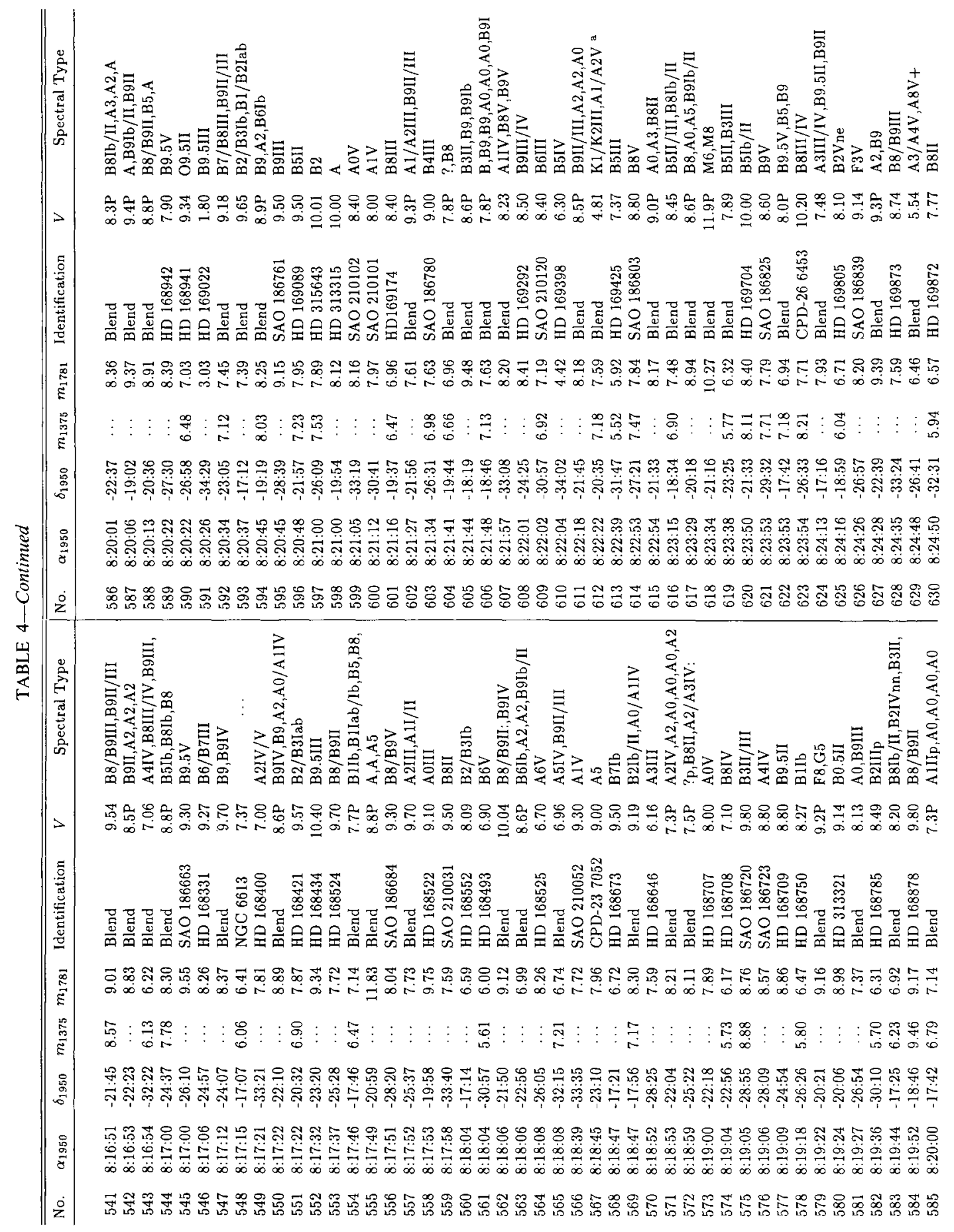




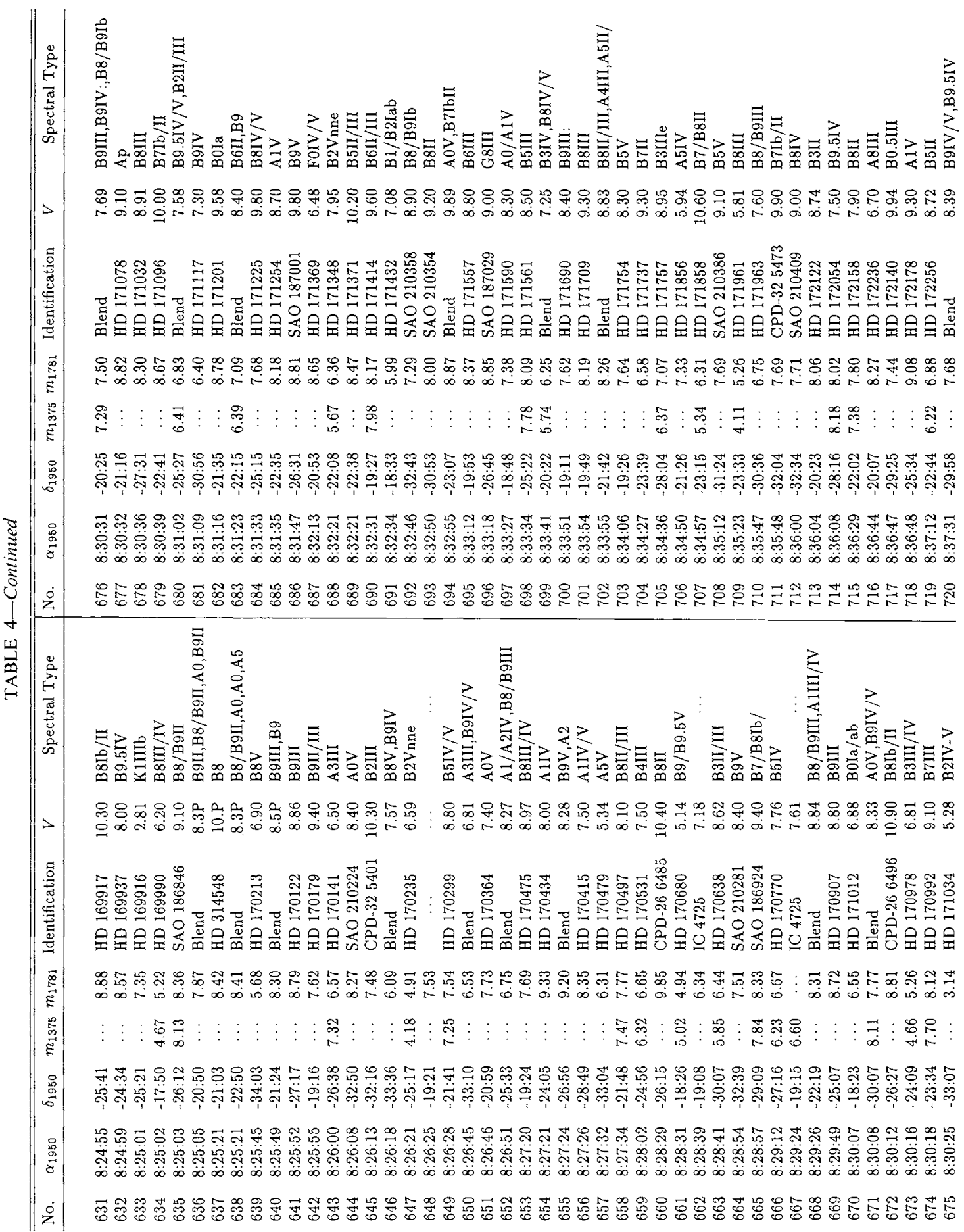


TABLE 4-Continued

\begin{tabular}{lcccclll}
\hline \hline No. & $\alpha_{1950}$ & $\delta_{1950}$ & $m_{1375}$ & $m_{1781}$ & Identification & $V$ & \multicolumn{1}{c}{ Spectral Type } \\
\hline & & & & & & & \\
721 & $8: 37: 38$ & $-21: 34$ & 6.89 & 7.56 & HD 172370 & 8.80 & B5III \\
722 & $8: 37: 57$ & $-31: 11$ & $\ldots$ & 7.29 & SAO 210457 & 8.80 & B5V \\
723 & $8: 38: 13$ & $-28: 56$ & 5.82 & 6.61 & Blend & 7.49 & B5II/III,B9.5V \\
724 & $8: 38: 46$ & $-23: 54$ & $\ldots$ & 7.84 & HD 172546 & 6.23 & A3m \\
725 & $8: 38: 46$ & $-27: 33$ & 5.82 & 6.52 & HD 172533 & 8.32 & B5III \\
726 & $8: 39: 04$ & $-23: 13$ & $\ldots$ & 8.10 & Blend & $8.6 \mathrm{P}$ & B8II,B7Ia,B7II \\
727 & $8: 39: 12$ & $-29: 42$ & $\ldots$ & 7.41 & SAO 187151 & 8.80 & B8II \\
728 & $8: 39: 17$ & $-20: 21$ & $\ldots$ & 7.25 & HD 172696 & 7.10 & Ap \\
729 & $8: 39: 31$ & $-22: 14$ & $\ldots$ & 7.64 & Blend & 8.18 & B5II/III,A0,A0IV/V \\
730 & $8: 39: 55$ & $-27: 45$ & $\ldots$ & 7.45 & SAO 187170 & 8.60 & B8IV \\
731 & $8: 40: 14$ & $-22: 29$ & $\ldots$ & 7.36 & HD 172854 & 7.71 & B3III \\
732 & $8: 40: 35$ & $-23: 19$ & $\ldots$ & 8.95 & HD 172906 & 9.30 & B9III \\
733 & $8: 41: 48$ & $-25: 06$ & 4.31 & 4.99 & HD 173117 & 5.83 & B8III \\
734 & $8: 42: 13$ & $-28: 18$ & $\ldots$ & 6.92 & Blend & 7.72 & B9IV,B8III \\
735 & $8: 42: 42$ & $-28: 54$ & $\ldots$ & 7.09 & Blend & 7.47 & B8/B9II,A0,A9 \\
736 & $8: 43: 04$ & $-27: 04$ & $\ldots$ & 2.72 & HD 173300 & 3.20 & B8III \\
737 & $8: 43: 46$ & $-23: 44$ & $\ldots$ & 8.30 & HD 173536 & 9.40 & B8Ib \\
738 & $8: 43: 49$ & $-22: 24$ & $\ldots$ & 7.38 & HD 173570 & 8.80 & B7III/IV \\
739 & $8: 43: 50$ & $-22: 41$ & $\ldots$ & 7.62 & HD 173571 & 8.80 & B5III \\
740 & $8: 43: 54$ & $-30: 08$ & $\ldots$ & 7.17 & HD 173502 & 9.74 & B1.5Ib \\
741 & $8: 44: 42$ & $-28: 27$ & $\ldots$ & 7.60 & HD 173657 & 7.30 & B9 \\
& & & & & & &
\end{tabular}

${ }^{\text {a }}$ Object 612 . Although the A star is about ten magnitudes fainter in $V$ than the $\mathrm{K}$ star, it may contribute a comparable amount to the ultraviolet flux.

NoTE.-Table 4 also appears in machine-readable form in the AAS CDROM Series, Vol. 4.

brighter than $m_{1781}$. Since a similar effect is not seen in the comparison with the ANS magnitudes, we conclude that it is caused by a departure from linearity in the $T D-1$ scale at the faint end. On the other hand, all three previous data sets tend to have brighter magnitudes at the bright end. We attribute this to uncertainties in our saturation correction.

Figure 4 compares $m_{1375}$ with magnitudes from $O A O 2$ and S201. While the $O A O 2$ bands bracket the present $\lambda 1375$ band, the $C$ magnitude from $\mathbf{S} 201$ approximates it closely. The $\mathbf{S} 201$ $L$ band extends to a shorter wavelength and includes the Ly $\alpha$ line of hydrogen.

Figures $4 a$ and $4 b$ show a linear relationship between our magnitude and the $O A O 2$ magnitudes for stars fainter than $m_{1375}=1$. The zero points are in close agreement (mean differences of 0.23 and 0.14 mag with rms scatter of 0.22 and $0.15 \mathrm{mag}$ for $m_{1330}$ and $m_{1430}$, respectively, omitting the four brightest stars). The S201 $C$ magnitudes (Fig. $4 c$ ) are fainter than ours by 0.50 (omitting four deviant points) with an rms scatter of 0.37 . There is also a suggestion of a scale difference in that the points in Figure $4 c$ seem to have a slope greater than unity; a least squares fit yields a slope of 1.113 . The $\$ 201 L$ magnitudes (Fig. $4 d$ ) are also fainter than ours on the average. However, there is more scatter and a significant number of the $S 201 L$ magnitudes are anomalously bright. This may be caused by contamination by Lyman- $\alpha$.

The sizable systematic difference between our magnitudes and the S201 magnitudes indicates a difference between the S201 calibration and the $I U E$ intensity scale which was used to calibrate the present photometry. This will be further discussed in another paper using the pre- and post-flight calibrations. For the present, we will assume that the $I U E$ calibration is to be preferred.
The scatter in the comparison of previous data with our photometry is the smallest for the $O A O 2$ magnitudes. Ignoring errors in the latter gives an upper limit for the internal errors of our magnitudes of between 0.15 and $0.29 \mathrm{mag}$. Since the S201 magnitudes and the present magnitudes were obtained with similar cameras and analyzed in a similar fashion, we might expect similar internal errors in the two data sets. Under this assumption, the internal standard deviation of the present photometry is $\sigma=0.37 / \sqrt{2}=0.26 \mathrm{mag}$. We can obtain another upper limit on the errors by noting that the color $\left(m_{1375}-\right.$ $m_{1781}$ ) is relatively insensitive to temperature (as shown in Fig. $1)$ and to interstellar absorption. The rms scatter of $\left(m_{1375}-\right.$ $m_{1781}$ ) is 0.38 . Assuming this is dominated by random errors in the magnitudes, the corresponding internal errors in the individual magnitudes are $\sigma=0.38 / \sqrt{2}=0.27 \mathrm{mag}$. These estimates agree well with what we found from the IUE spectra above. We will adopt $0.25 \mathrm{mag}$ as a preliminary estimate of the internal uncertainty of our ultraviolet photometry.

There is another set of images which covers the Galactic center and overlaps the fields being studied here. When reduced they will provide another check on both the internal errors and the absolute calibration of our data.

\section{DISCUSSION}

For the following discussion we have selected only the ultraviolet objects for which data from SIMBAD can be used. This requires that the object be identified with a unique star. Further, that star must have a spectral type and subtype and a $V$ magnitude. The existing data for these fields (which have both been covered by the Michigan Spectral Survey; Houk 1978, 1982; Houk \& Smith-Moore 1988) is extensive and our selected sample contains 727 stars (or $57 \%$ of the total). In fact, MK spectral types exist for most of the selected sample.

Figure 5 presents histograms of the ultraviolet magnitudes of all the objects in Tables 4 and 5. The sudden drop in the distributions at the faint end indicates limiting magnitudes between 8.5 and 9.0 in the M8 field but about 8.0 for the $\zeta$ Sco images. The different limits for the two fields are consistent with the exposure times. For both fields the distributions begin to flatten out fainter than about seventh magnitude. This probably reflects incompleteness in our sample due to crowding.

In Figure 6 we plot a histogram of the spectral types of the stars in the selected sample. We have not included ten Ap stars, two WC stars and one star of each of the types WNn, DA, Am, Asp and $\mathrm{O} / \mathrm{Be}$. As was the case in Monoceros and Orion (Papers I and II), the most common type of star is near spectral type A0. However, there are many more early B stars than in the previous studies; stars earlier than B 6 make up 50\% of the stars in the $\zeta$ Sco field and $40 \%$ of those in the M8 field but only $12 \%$ and $20 \%$ of the Monoceros and Orion samples. The presence of a number of $\mathrm{OB}$ associations in these fields (Ara $\mathrm{OB} 1$ and Sco OB1 in the $\zeta$ Sco field and Sgr OB1, Sgr OB4, Sgr OB7, and portions of Sgr OB6 and Ser OB1 in the M8 field) might account for this. On the other hand, stars with spectral types of F0 and later comprise only $1 \%$ of the present objects while they were $21 \%$ and $6 \%$ of the Monoceros and Orion samples. 


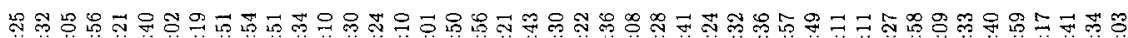

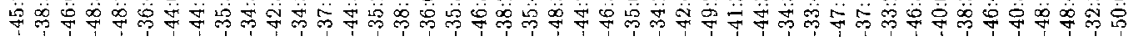

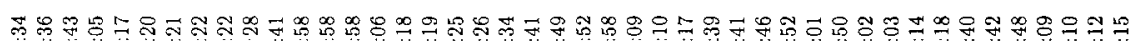

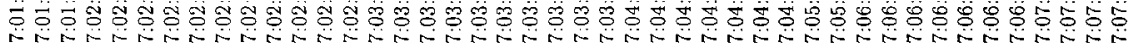

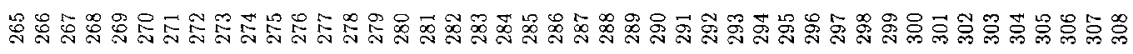


TABLE 5-Continued

\begin{tabular}{lcccclcl}
\hline \hline No. & $\alpha_{1950}$ & $\delta_{1950}$ & $m_{1375}$ & $m_{1781}$ & Identification & $V$ & Spectral Type \\
\hline & & & & & & & \\
529 & $7: 42: 50$ & $-39: 24$ & $\ldots$ & 5.59 & HD 161277 & 7.08 & B9 \\
530 & $7: 43: 03$ & $-40: 40$ & $\ldots$ & 7.40 & HD 161310 & 8.30 & A1V \\
531 & $7: 43: 14$ & $-43: 29$ & 5.43 & 5.64 & HD 161312 & 7.64 & B6II/III \\
532 & $7: 43: 16$ & $-42: 29$ & 7.95 & 7.59 & CD 4212464 & 9.00 & B9II \\
533 & $7: 43: 30$ & $-40: 58$ & $\ldots$ & 5.55 & HD 324325 & $10 . P$ & A0 \\
534 & $7: 43: 32$ & $-40: 49$ & 5.35 & $\ldots$ & Blend & 7.56 & B8II,B3III \\
535 & $7: 43: 38$ & $-42: 02$ & 7.24 & 7.51 & Blend & $8.3 P$ & B9V,A0 \\
536 & $7: 44: 15$ & $-41: 04$ & $\ldots$ & 5.74 & CD-41 12036 & 9.9 & A9V \\
537 & $7: 44: 18$ & $-40: 55$ & 5.66 & $\ldots$ & HD 161531 & 9.06 & B2/B3Ib \\
538 & $7: 44: 26$ & $-42: 12$ & 7.63 & $\ldots$ & HD 324369 & 9.99 & B2 \\
539 & $7: 44: 32$ & $-42: 32$ & 7.51 & 7.21 & CD-42 12487 & 8.90 & B9III \\
540 & $7: 44: 59$ & $-42: 56$ & 7.40 & $\ldots$ & Blend & $8.4 P$ & B8/B9Ib,A2 \\
541 & $7: 45: 58$ & $-42: 25$ & 7.43 & $\ldots$ & Blend & $8.9 P$ & B4II/III,A2
\end{tabular}

${ }^{a}$ Object 32. This object coincides with the G5V star HD 147513 with $V=5.40$ and the white dwarf CD-38 10980 with $V=10.98$. McCook \& Sion 1987 give a spectral type of DA2, corresponding to an effective temperature of about 25,000 for the white dwarf. We have tabulated the white dwarf's $V$ magnitude since it should dominant the ultraviolet flux.

${ }^{b}$ Object 316 . Although the A1 V star HD 155237 is six magnitudes fainter in $V$ than the F3 III-IV star HD 155203, the contributions of the two in the ultraviolet may be comparable due to the temperature difference (ignoring interstellar extinction).

NOTE.-Table 5 also appears in machine-readable form in the AAS CD-ROM Series, Vol. 4.

Figure 7 shows plots of the color excesses $E_{17, V}=\left(m_{1781}-\right.$ $V)-\left(m_{1781}-V\right)_{0}$ and $E_{13, V}=\left(m_{1375}-V\right)-\left(m_{1375}-V\right)_{0}$ against the apparent distance modulus, $\mu_{V}=V-M_{V}$. Only stars with spectral classes of $\mathrm{O}, \mathrm{B}, \mathrm{A} 0$ and $\mathrm{A} 1$ and with luminosity classes were included (for a total of 566 different stars plotted in Fig. $7 a, 7 b$, or both ). The absolute magnitudes, $M_{V}$, were obtained from the spectral types and luminosity classes using the tables given by Lang (1992). Lang's tables of effective temperature and gravity as a function of spectral type and luminosity class were used together with Tables 1 and 2 to obtain the intrinsic colors, $\left(m_{1781}-V\right)_{0}$ and $\left(m_{1375}-V\right)_{0}$.

The wavelength dependence of ultraviolet extinction varies considerably from one line of sight to another. Mathis (1990) has tabulated the extinction law for cases with $R=A_{V} / E_{B-V}=$ 3.1 and 5.0. The mean visual extinction was estimated by Sharov (1964) to range from $\rho_{V} \sim 0.6 \mathrm{mag} \mathrm{kpc}-1$ to $\rho_{V} \sim 3.5$ mag kpc ${ }^{-1}$ over our fields. In Figure 7 we have plotted the run of color excess with apparent distance modulus for four combinations of $R$ and $\rho_{V}$. We will regard these as covering the extremes we might encounter here.

The increasing scatter at larger apparent distance modulus indicates that the mean reddening does indeed range as widely as Sharov's study suggested. However, it appears that variations in the reddening law are also required to fully account for the distribution. Since many of the early-type stars in these fields suffer high extinction, variations in the ultraviolet extinction curve could also contribute to scatter in the measured ultraviolet magnitudes as a function of spectral type, $V$ or $E(B-$ $V$ ). The detailed implications of the ultraviolet photometry for interstellar extinction will be discussed after we have extracted the photometry for other fields in the far-ultraviolet survey.

While a majority of points fall between the extreme absorption lines in Figure 7, there are many which do not. The stars below the lower curve probably suffer extinction which is higher than the average. On the other hand, stars above the upper curve are more numerous and can not be explained as easily.

An examination of Figure 7 shows that raising the upper curves $(0.6,5.0)$ by about 0.5 magnitudes would encompass the nearest and presumably least reddened of the stars. Such an adjustment could be accomplished, for example, by altering the adopted effective temperatures by an amount corresponding to one or one and a half spectral subclasses. Such an uncertainty in the temperature scale or in the calculated colors is plausable. Alternatively, if we adopted the ultraviolet magnitude scale suggested by the comparison with the S201 data (discussed in $\S 4$, above), the points of individual stars in Figure 7 would move down by 0.5 magnitudes. While this, too, would alleviate the discrepancy, we prefer the present calibration until the pre- and post-flight calibrations have been analyzed.

Based on the discussion of errors at the end of $\S 4$, we estimate that the uncertainty in the color excesses is about 0.50 magnitudes at a 1.4 sigma level. The dashed lines in Figure 7 show the position of the $(0.6,5.0)$ curve adjusted upward by one magnitude to include both uncertainties. The stars above the dashed curves are likely to have ultraviolet excesses. However, to be conservative, we have only included stars with measurements of both ultraviolet magnitudes and for which both measurements place the star above the dashed line in our final list of stars with ultraviolet excesses. The 25 objects meeting these criteria (which constitute $7 \%$ of the 341 stars in Figure 7 which have both magnitudes) are indicated by open circles and are listed in Table 6. It should be kept in mind that many of the objects which failed to meet our requirement for excesses in both colors may nonetheless actually have an excess. Additionally, many of the objects not included in Figure 7 (for example stars deemed to be blends) would no doubt exhibit an excess if examined carefully. Thus, the stars in Table 6 represent a very conservative lower limit to the true incidence of ultraviolet excesses.

In Papers I and II a small number of stars were found with apparent ultraviolet excesses. We suggested that these objects were nearby hot white dwarfs. The availability of MK spectral types for the current sample changes this picture considerably. The spectral types in Table 6 range from B1 to A2 (our arbitrary cutoff) and the luminosity classes range from $\mathrm{V}$ to $\mathrm{Ib}$. Clearly these are not nearby white dwarfs.

There are several ways to explain the stars with ultraviolet excesses. One possibility is that the ultraviolet magnitudes of these stars (which represent slightly less than $5 \%$ of the objects in Fig. 7) are incorrect. However, $m_{1781}$ and $m_{1375}$ were obtained with two different cameras; any errors due to camera or emulsion artifacts should be uncorrelated between them. Thus, our requirement that the excess be evident in both magnitudes at a statistically significant level should eliminate any stars with erroneously bright ultraviolet magnitudes.

Given the spatial resolution of our images, we must consider the possibility that the excess ultraviolet emission arises from the chance superposition of another object on the star identified as the ultraviolet object. For example, a foreground hot white dwarf could easily explain the ultraviolet brightness. To 

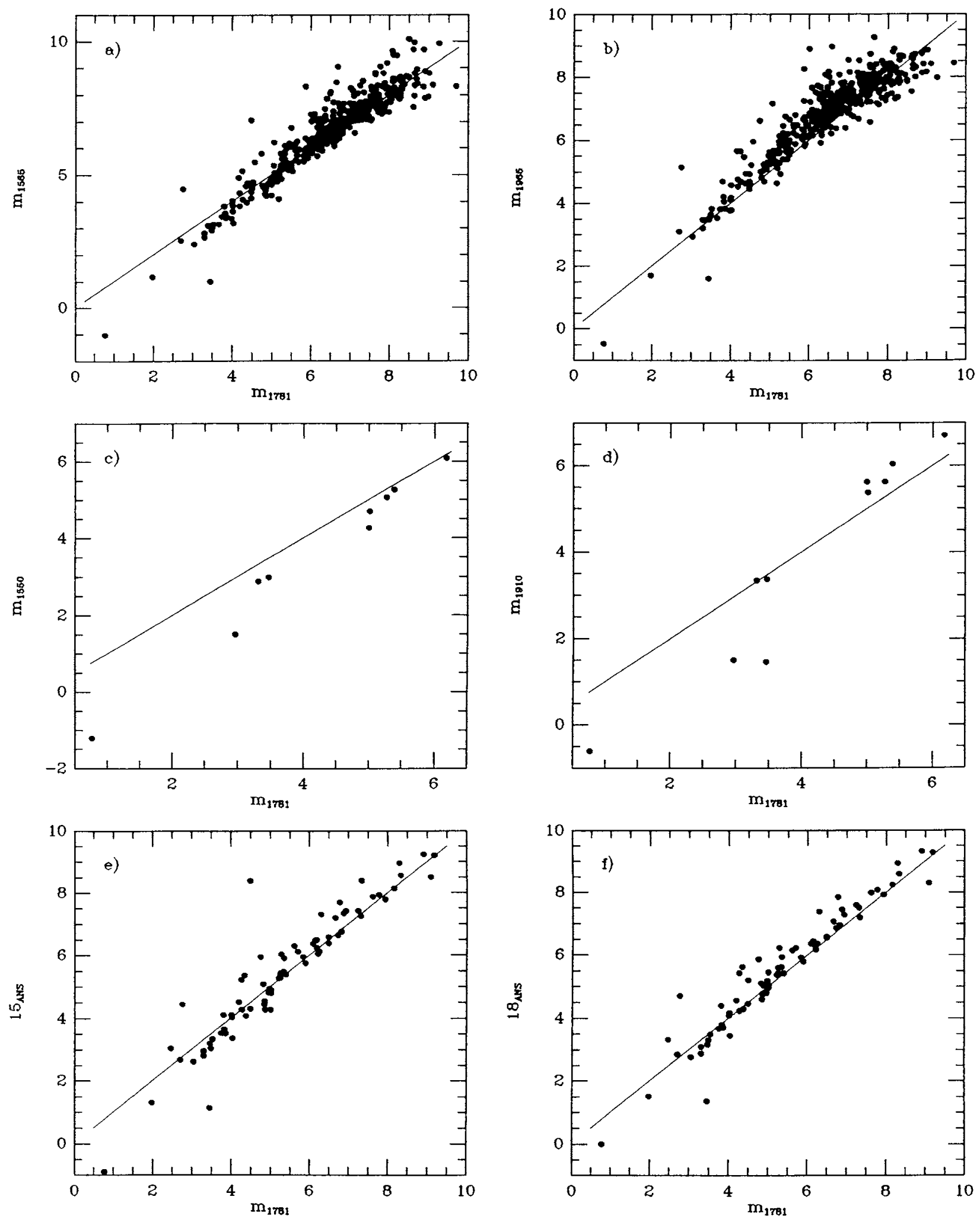

FIG. 3.-Comparisons of $m_{1781}$ with data from the $T D-1(a$ and $b), O A O 2(c$ and $d)$ and $A N S(e$ and $f)$ satellites 

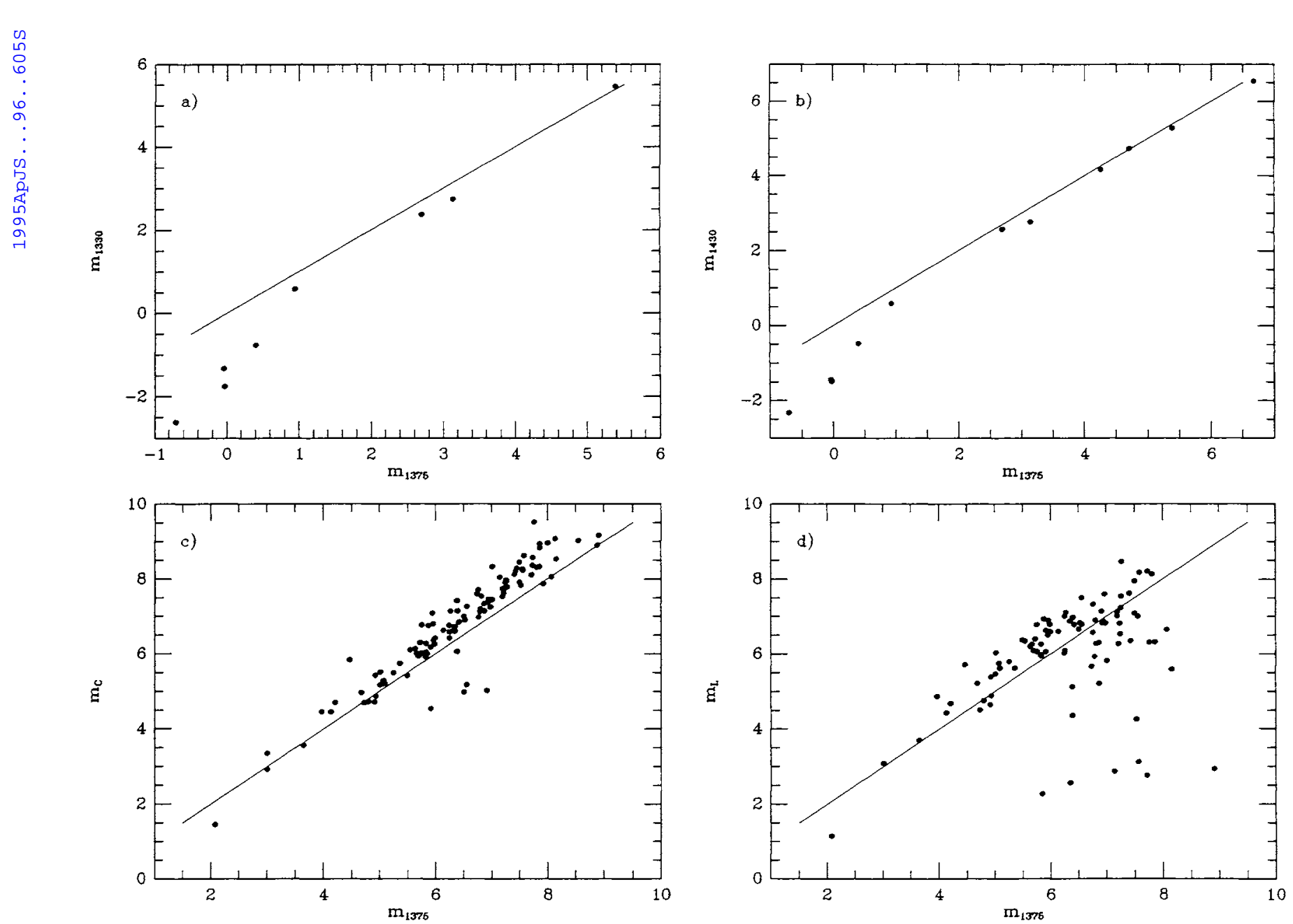

FIG. 4.-Comparison of $m_{1375}$ with $O A O 2$ and $\$ 201$ magnitudes
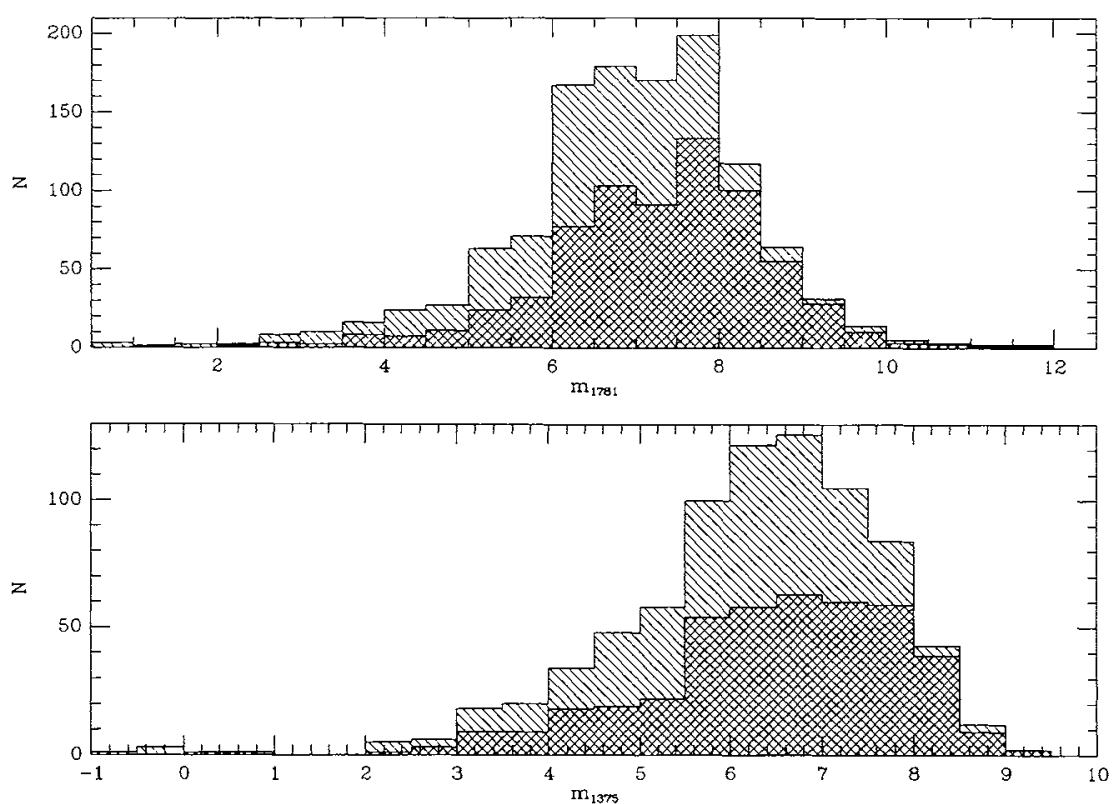

FIG. 5.-Frequency distribution of stars with $m_{1781}(a)$ and $m_{1375}(b)$. The more darkly shadded regions represent the M8 sample while the lightly shaded region represents the $\zeta$ Sco stars. 


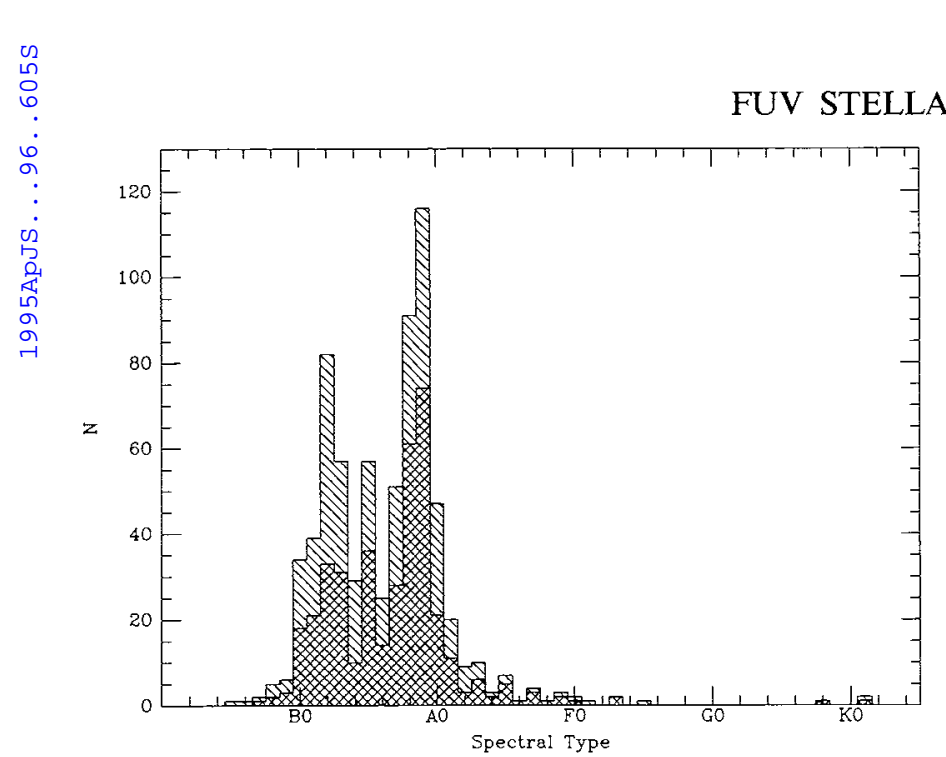

FIG. 6.-Frequency distribution of stars in the selected sample with spectral type. The more darkly shaded portion of the histogram represents the M8 sample while the lightly shaded region represents the $\zeta$ Sco stars.
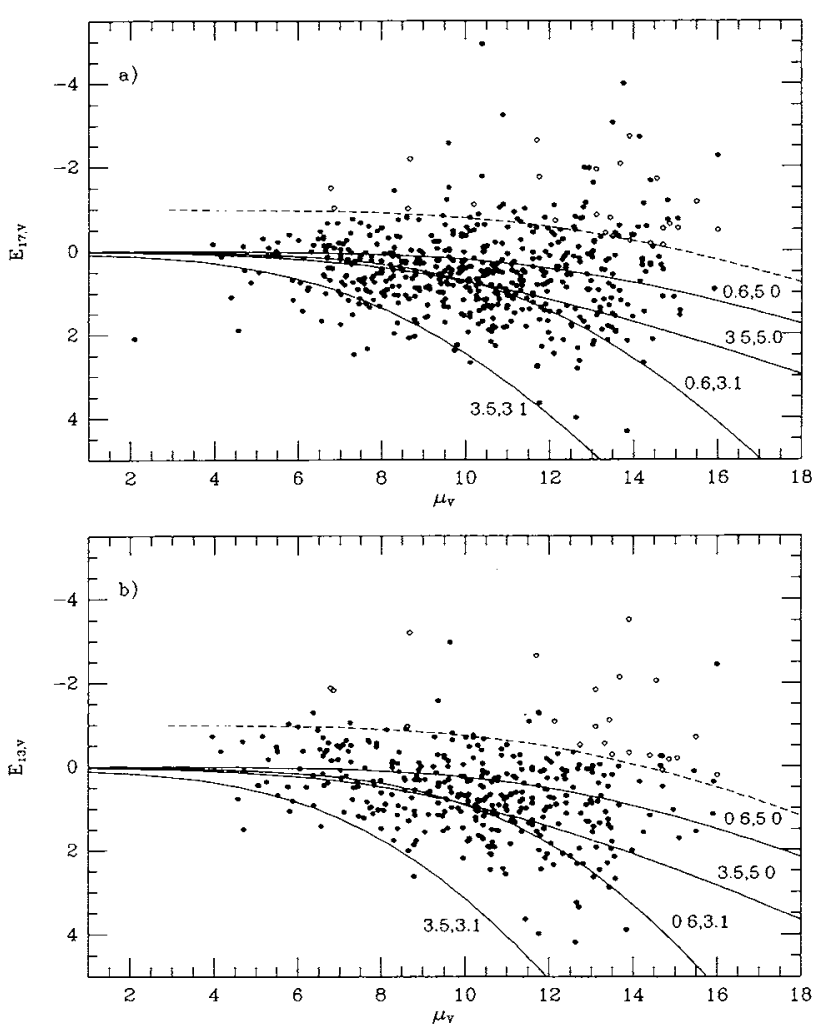

FIG. 7.-Plots of the color excesses in $\left(m_{1781}-V\right)$, denoted by $E_{17, V}$, and $\left(m_{1375}-V\right)$, denoted by $E_{13, V}$, against apparent visual distance modulus, $\mu_{V}=V-M_{V}$, for stars with $\mathrm{MK}$ spectral types of $\mathrm{O}, \mathrm{B}, \mathrm{A} 0$, and $\mathrm{Al}$ and known luminosity class. Open circles denote stars with significant ultraviolet excesses as discussed in the text. The solid curves show the expected variation of color excess with apparent distance modulus under various assumptions. Each is labeled with the $V$ absorption per kiloparsec, $\rho v$ (either 0.6 or 3.5 ) and the ratio of total to selective absorption, $R=A_{V} /$ $E_{B-V}$ (either 3.1 or 5 ) for the assumed absorption law. The dashed lines are the curves for $R=5$ and $\rho v=0.6$ mag kpc ${ }^{-1}$ shifted upward by 1 magnitude as explained in the text.
TABLE 6

StaRs Which EXHIBIT Ultraviolet EXCESSES

\begin{tabular}{lllrrrr}
\hline \hline No. & Identification & Sp. Type & \multicolumn{3}{c}{$\mu_{V}$} & \multicolumn{4}{c}{$E_{17, V}$} & $E_{13, V}$ & $E_{13,17}$ \\
\hline$\zeta 251$ & HD 153767 & A0V & 6.8 & -1.5 & -1.9 & -0.4 \\
$\zeta$ 287 & HD 154410 & A0V & 6.8 & -1.0 & -1.8 & -0.8 \\
$\zeta$ 409 & HD 157698 & B7III & 8.6 & -1.0 & -1.0 & 0.0 \\
M 200 & HD 164295 & B9.5V & 8.7 & -2.2 & -3.2 & -1.0 \\
M 155 & SAO 186016 & B9III & 10.2 & -1.1 & -0.7 & 0.4 \\
$\zeta 221$ & CPD-29 4400 & B9.5 III & 11.7 & -2.6 & -2.6 & -0.0 \\
$\zeta 518$ & HD 160856 & B6III & 11.8 & -1.8 & -1.3 & 0.5 \\
$\zeta 463$ & HD 159041 & B9Ib/II & 12.1 & -0.7 & -1.1 & -0.4 \\
$\zeta$ 239 & SAO 208310 & B4II/III & 12.7 & -0.8 & -0.5 & 0.2 \\
M 178 & HD 164030 & B5III & 13.1 & -1.9 & -1.8 & 0.1 \\
M 443 & SAO 186514 & B7II & 13.1 & -0.8 & -1.0 & -0.1 \\
M 357 & HD 166192 & B2II & 13.3 & -0.4 & -0.6 & -0.1 \\
M 272 & HD 165132 & B5/B6Ib & 13.4 & -0.8 & -1.1 & -0.3 \\
M 463 & HD 167333 & B4III & 13.5 & -0.3 & -0.3 & 0.0 \\
M 194 & CPD-31 5209 & B8/B9II & 13.7 & -2.1 & -2.1 & -0.1 \\
M 707 & HD 171858 & B7/B8II & 13.9 & -2.7 & -3.5 & -0.8 \\
M 317 & HD 165765 & B2III & 13.9 & -0.2 & -0.3 & -0.1 \\
$\zeta 495$ & CD-41 11898 & B8Ib & 14.4 & -0.2 & -0.2 & -0.1 \\
M 505 & CPD-29 5474 & B8Ib/II & 14.6 & -1.7 & -2.1 & -0.4 \\
M 408 & CPD-31 5415 & B7Ib/II & 14.7 & -0.5 & -0.2 & 0.3 \\
$\zeta 485$ & HD 159767 & B7Ib & 14.7 & -0.1 & 0.1 & 0.2 \\
$\zeta 275$ & CD-42 11823 & B7/B8Ib & 14.8 & -0.6 & -0.2 & 0.5 \\
M 379 & CPD-31 5395 & B4II & 15.1 & -0.5 & -0.2 & 0.3 \\
M 354 & CPD-32 5172 & B5Ib/II & 15.5 & -1.1 & -0.7 & 0.5 \\
M 159 & CPD-30 5098 & B2/B3Ib & 16.0 & -0.5 & 0.2 & 0.7 \\
\hline
\end{tabular}

assess this idea we interrogated the SIMBAD data base for objects within $10^{\prime}$ of 225 locations scattered across our fields. A $10^{\prime}$ radius for identifying superposed objects is rather conservative; stars separated by that much on the sky would most likely be recognized as two objects in our extraction of stellar magnitudes. Fifty percent of the random locations yielded one or more objects which would be deemed plausible sources of far-ultraviolet radiation (i.e., earlier than A2 in spectral type). Thus, if the 25 objects in Table 6 were due to chance superpositions, there should be an approximately equal number of other ultraviolet objects superposed on locations without a plausible ultraviolet source from SIMBAD. Tables 4 and 5 contain only eight objects with no associated SIMBAD object and another nine where the identified object was too late in spectral type to be the source of the ultraviolet radiation. While this total of 17 objects is not significantly fewer than the objects listed in Table 6, as noted above, the number of stars in the table represents a lower limit to the incidence of ultraviolet excesses. Thus, we conclude that the source of the excess ultraviolet in many or most of the objects in Table 6 is physically related to the identified stars.

Is it possible that the ultraviolet radiation comes from a companion to the visible star? For this to be the case the companion would have to be fainter than the primary star in the visible but enough hotter to dominate the ultraviolet spectrum. For most of the stars in Table 6 a suitable companion can not be found among normal stars. Even very hot white dwarfs are simply too faint in the ultraviolet to outshine B or early A dwarfs. The only possibility which seems marginally feasible is to combine one of the later giants in the table with a hotter main sequence star. However, this could only explain a very few of the stars in Table 6 .

The stars in Table 6 should be further investigated to determine the source of the ultraviolet excesses. Investigation of the objects with no optical identification and those identified with 
late stars would also be worthwhile. It is likely that some interesting objects will be found in that way.

We thank B. C. Dohne and Brian Dorland for assistance in the data analysis. We also thank many coworkers at the Naval Research Laboratory and elsewhere who assisted in or contributed to the development and flight of the far-ultraviolet cameras on STS-39. The flight opportunity and mission support were provided by the Air Force Space Test Program. The in- strument development was sponsored by the Office of Naval Research. Data needed for this research were obtained from the IUE Regional Data Analysis Facility and the Astronomical Data Center which are operated by NASA at the Goddard Space Flight Center and the SIMBAD database which is operated by CDS in Strasbourg, France. Much of the data analysis was performed at NRL's Backgrounds Data Center. The use of these facilities made an important contribution to this project and is gratefully acknowledged.

\section{REFERENCES}

Carruthers, G. R. 1986, Proc. SPIE, 687, 11

Carruthers, G. R., Dohne, B. C., Shephard, K. K., Christensen, S. A., \& Schmidt, E. G. 1994, in preparation

Carruthers, G. R., Morrill, J. S., Dohne, B. C., \& Christensen, S. A. 1992, Proc. SPIE, 1764, 21

Carruthers, G. R., \& Page, T. 1983, ApJS, 53, 623

- 1984a, ApJS, 54, 271

.1984b, ApJS, 55, 101

1984c, PASP, 96, 447

Christensen, S. A., Carruthers, G. R., \& Dohne, B. C. 1994, in preparation

Code, A. D., Holm, A. V., \& Bottemiller, R. L. 1980, ApJS, 43, 501

Houk, N. 1978, The Michigan Catalogue of the Two-Dimensional Spectral Types for the HD Stars, Vol. 2 (Ann Arbor: Univ. Michigan)

. 1982, The Michigan Catalogue of the Two-Dimensional Spectral Types for the HD Stars, Vol. 3 (Ann Arbor: Univ, Michigan)

Houk, N., \& Smith-Moore, M. 1988, The Michigan Catalogue of the TwoDimensional Spectral Types for the HD Stars, Vol. 4 (Ann Arbor: Univ. Michigan).
Kurucz, R. L. 1993, in Improvements in Light Curve Modeling of Eclipsing Binary Stars, ed. E. F. Milone (Berlin: Springer), 93

Lang, K. R. 1992, Astrophysical Data: Planets and Stars (New York: Springer)

Mathis, J. 1990 ARA\&A, 28,37

Page, T., Carruthers, G. R., \& Heckathorn, H. M. 1982, Revised S201 Catalogue of Far-Ultraviolet Objects, NRL Report 8487 (Washington: Naval Research Laboratory)

Schmidt, E. G., \& Carruthers, G. R. 1993a, ApJ, 408,484, (Paper I) 1993b, ApJS, 89,259, (Paper II)

Sharov, A. S. 1964, Soviet Astron., 7,689

Stetson, P. B. 1987, PASP, 99, 191

Thompson, G. I., Nandy, K., Jumar, C. Monfils, A., Houriaux, L., Carnochon, A., \& Wilson, R. 1978, Catalogue of Stellar Ultraviolet Fluxes (London: Science Research Council)

Wesseluis, P. R., van Duinen, R. J., de Jong, A. R. W., Aalders, J. W. G., Luinge, W., \& Wildeman, K. J. 1982, A\&AS49, 427 


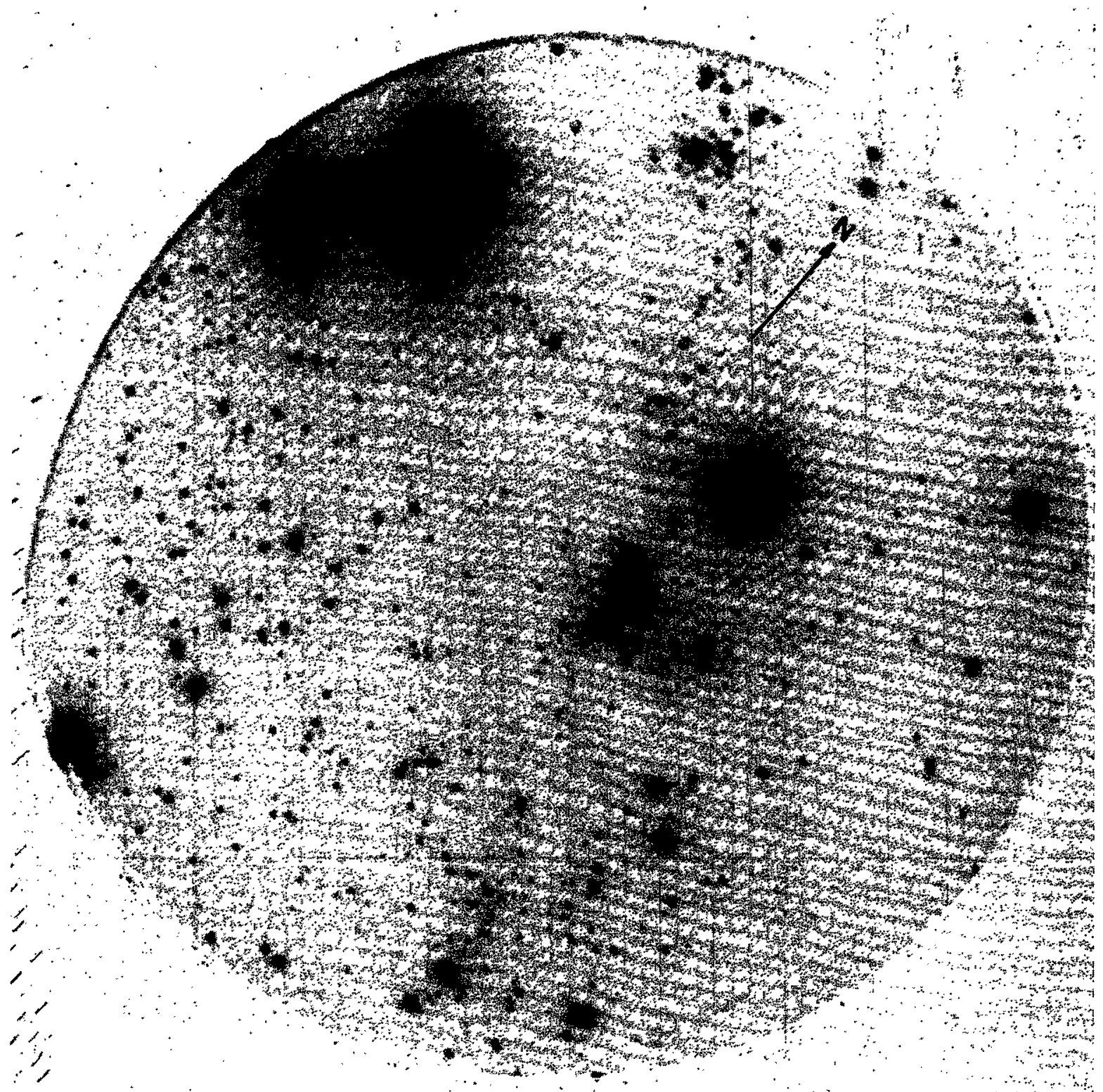

FIG. $2 a$

FIG. 2.-Prints of far-UV images of the two fields: $(a) \zeta$ Scorpii field, Camera $1\left(\lambda_{\text {eff }}=1375 \AA\right), 100$ second exposure; $(b) \zeta$ Scorpii field, Camera 2 $\left(\lambda_{\text {eff }}=1781 \AA\right), 30$ second exposure; $(c)$ Messier 8 field, Camera 1, 100 second exposure; $(d)$ Messier 8 field, Camera 2, 100 second exposure.

SCHMIDT \& CARRUTHERS ( 


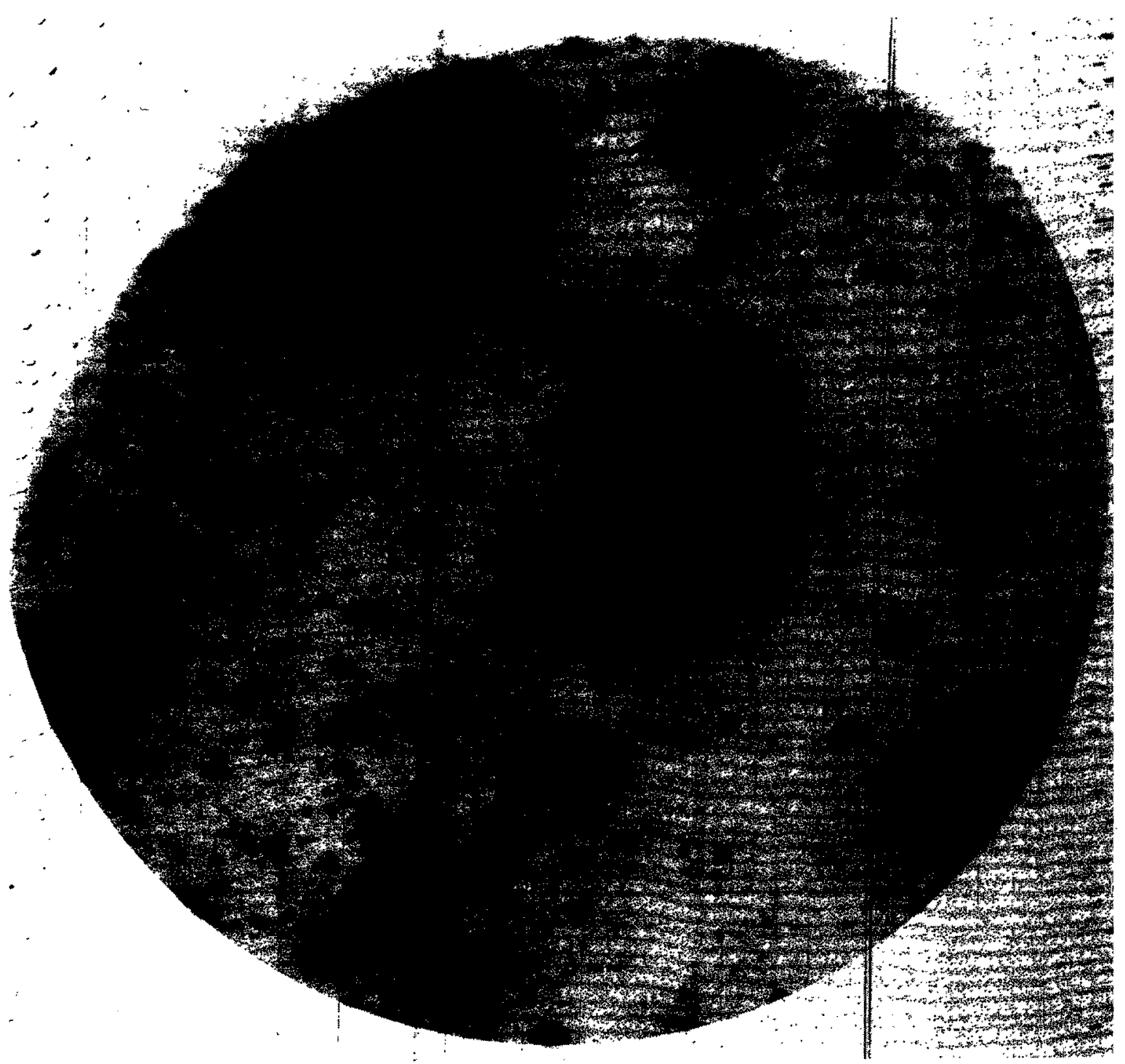

FIG. $2 b$

SCHMIDT \& CARRUTHERS ( see 96,605) 
PLATE 16

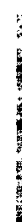

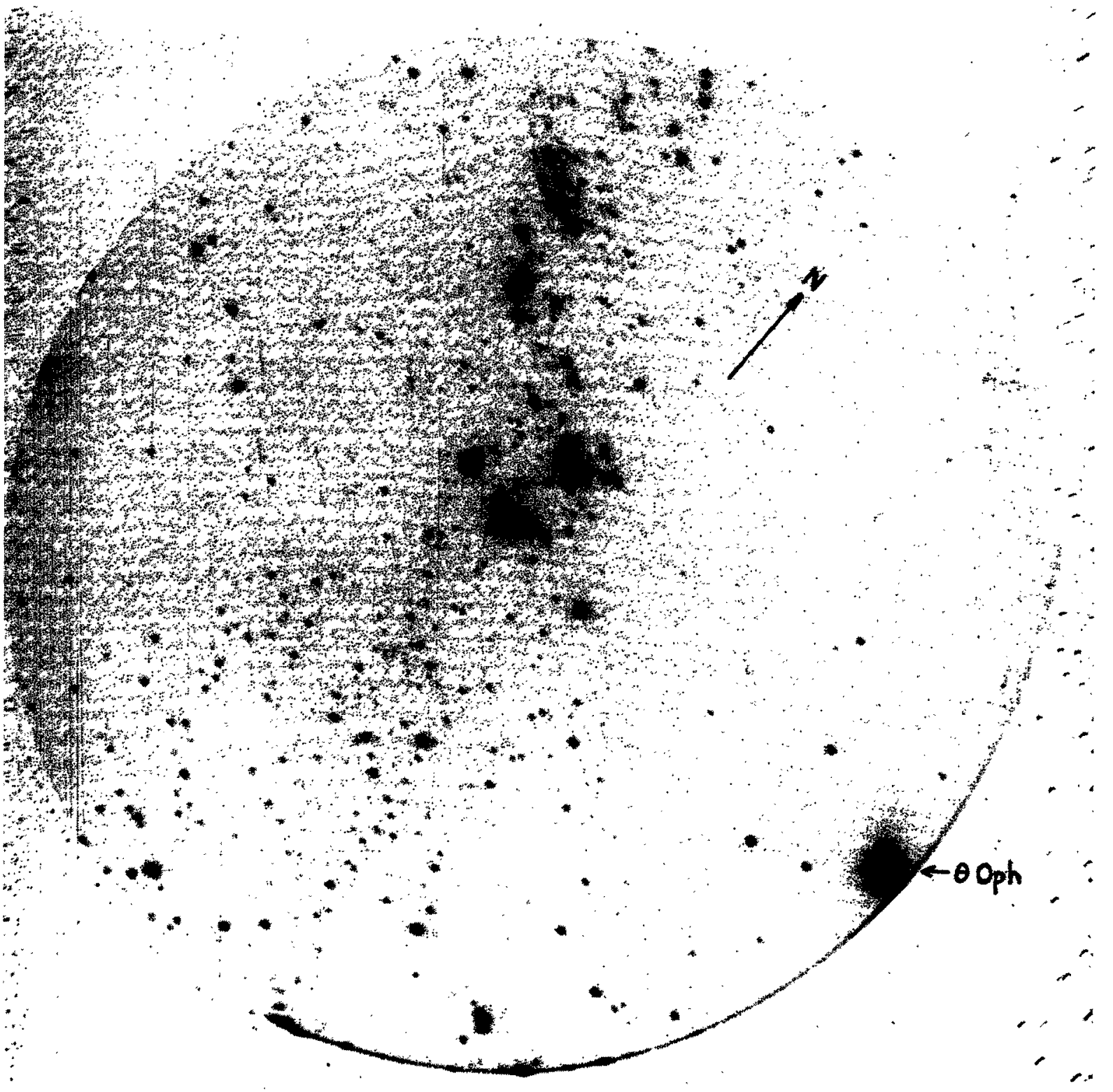

FIG. $2 c$

SCHMIDT \& CARRUTHERS ( see 96,605 ) 


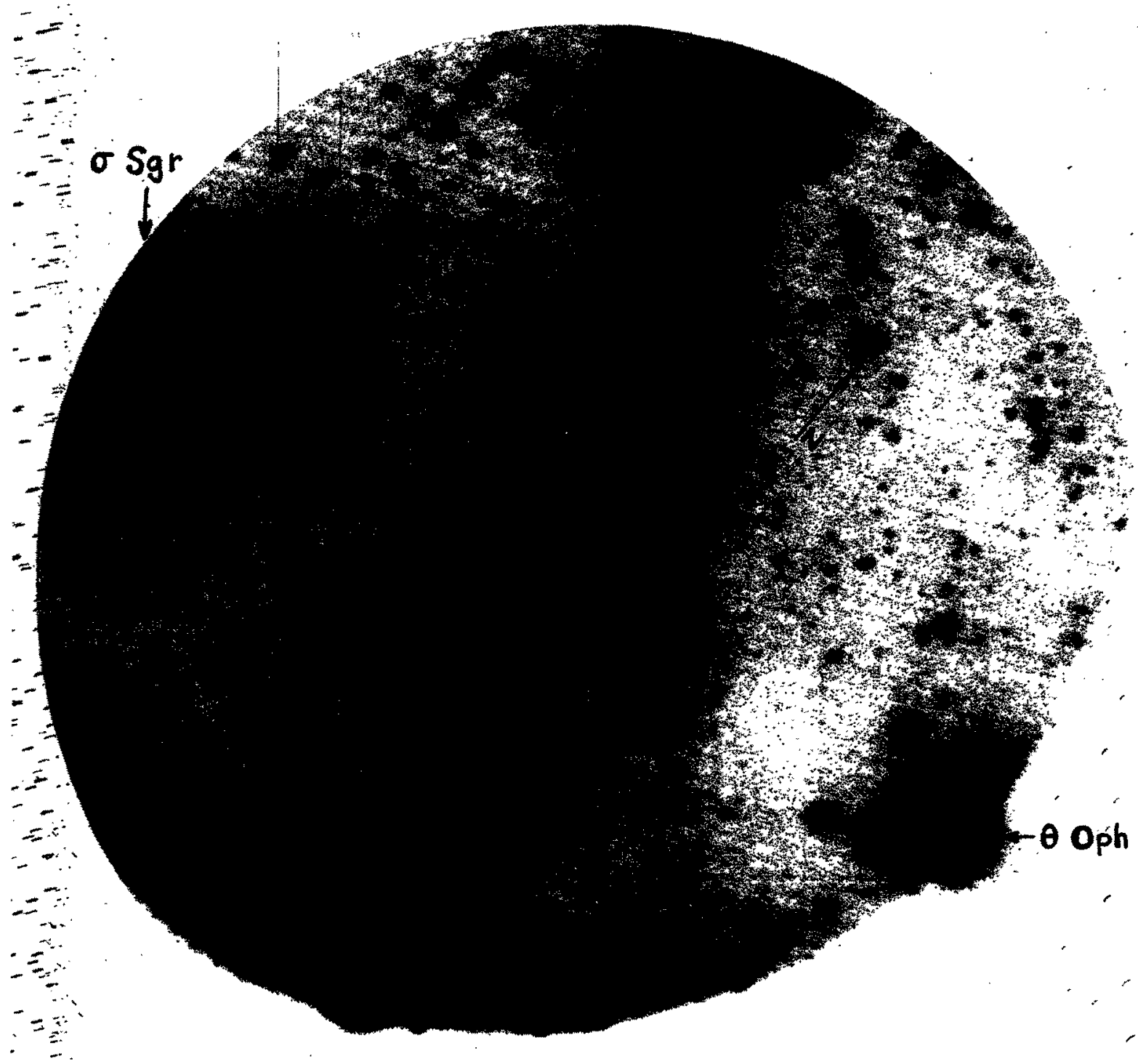

FlG. $2 d$

SCHMIDT \& CARRUTHERS ( see 96,605 ) 